\title{
PREDICATE CALCULUS FEATURE GENERATION
}

\author{
David Rothenberg \\ University College, Rutgers University
}

\begin{abstract}
A summary description (proof of theorems omitted): An adaptive pattern representation and recognition strategy for application to mechanized interpretation of (sampled) pictorial data (other applications are appropriate) is described. The system generates its own features which are formulae in a subset of the weak (in the sense that only quantification over finite sets is permitted) second order predicate calculus. The models of $s$ uch formulae define the "objects" in a description of the data, which is hierarchical both with respect to features and extensions. The hierarchy is automatically constructed, thereby implementing changes in "problem representation". Relations between the syntax and semantics of formulae in the weak second order predicate calculus are derived (by extending the syntax of the calculus) and utilized. Minimal use is made of the finiteness of the input data by the methods employed. That is, in pictorial pattern recognitions, the adaptive feature generation (i.e., "learning") algorithms are independent of the fineness of grain of the sampling of an input picture. Because this approach is used, many difficult problems of a purely mathematical nature acquire practical importance. Computation is reduced through the use of topological methods and the system is at present in a stage of development appropriate for programming and use in a variety of practical applications.
\end{abstract}

\section{The Problem}

The problem definition used conforms well to the theoretical framework proposed by Banerji (1969, 9-16, 103-175):

From a finite set of points, $\mathrm{U}=\left\{\mathrm{x}_{1}, \mathrm{x}_{2}, \ldots, \mathrm{x}_{\mathrm{n}}\right\}$, two ${ }^{1}$ disjoint collections, $\overline{\mathrm{K}}$ and $\overline{\mathrm{C}}$, of given subsets of $U$ (called regions) are given. That is, if " $R_{i}$ " denotes a region in $\bar{K}$ and " $R_{j}$ " a region in $\overline{\mathrm{C}}$,

$$
\begin{aligned}
& \bar{K}=\left\{R_{1}, R_{2}, \ldots, R_{n}\right\} \\
& \bar{C}=\left\{R_{N+1}, R_{N+2}, \ldots, R_{N+M}\right\} \\
& \forall i \forall j\left(R_{i} \neq R_{j}\right) \text { when } R_{i} \in \bar{K} \text { and } R_{j} \in \bar{C} .
\end{aligned}
$$

A formula in the weak second order predicate calculus, $\bar{P}(A)$, (called an ideal predicate or ideal feature) must be generated which is strongly satisfied by all regions $R_{i}$ in $\bar{K}$, and by no region $R_{j}$ in $\bar{C}$. We define a formula as being strongly satisfied by a region if it is satisfied by that region and is a proper subset of no other region which satisfies the

${ }^{1}$ This, of course, is easily generalized to $\mathrm{n}$ distinct collections. 
formula. (Of course, a feature can have only one free (set) variable ("A" in " $\overline{\mathrm{P}}(\mathrm{A})$ ").) Trivially, such a formula can be a disjunction of the conjunction of (the memberships of) all points of U (i.e., "constants") in each set in the collection $\overline{\mathrm{K}}$. However, the "shortest" possible formula (Banerji, 1969, 103-104) is sought: From a given set of unary and n-ary atomic predicates (all sequences of points in $U$ which satisfy each of these is given), a formula is constructed in which both the number of constants (specific points in U) and variables (both point and set) are minimal. (This definition of "shortness" somewhat resembles those developed by Kolmogorev and discussed in Löfgren (1967) and Solmonoff (1964a,b).) Such a substitution of a formula containing quantified variables for a formula containing a large number of constants (but no quantified variables) tends to result in the satisfaction of the formula by sets not in any of the collections. Hence, the formula is said to generalize (Banerji, 1969; 104, 157, 168, 175) the expression which involves only constants.

Actually the predicate calculus syntax is here extended to include a modified quantifier, $\bar{Q}^{(k)} \mathrm{x}$, which may be read, "for $\mathrm{k} \cdot 100$ percent of $\mathrm{x}^{\text {" (similarly to the reading of }}$ " $\forall \mathrm{x} "$ as "for all $\mathrm{x} "$ and " $\exists \mathrm{x}$ " as "there exists an $\mathrm{x} ")$. The degree of satisfaction of a set, $R_{n} \subset U$, with respect to a feature $P(A)$, when $R_{n}$ does not satisfy $P(A)$, is defined as -1 plus the largest value of $k$ in all replacements of a single universal quantifier by $\bar{Q}^{(k)}$ in $P(A)$ such that $R_{n}$ satisfies $P(A)$ as a consequence of that replacement. When $R_{n}$ satisfies $P(A)$, the degree of satisfaction is the largest value of $k$ in all replacements of a single existential quantifier by $\bar{Q}^{(k)}$ such that $R_{n}$ still satisfies $P(A)$. A topology for the space, $U$, is developed so that $U$ can be mapped into a space, $S$, with fewer points, in such fashion that the resulting loss of relevant information is bounded. That is, each $R_{n} \subset U$ has a "degraded image", $\bar{R}_{n} \subset S$, such that if $R_{n}$ satisfies $P(A)$, then the degree of satisfaction of $\bar{R}_{n}$ with respect to $P(A)$ differs from that of $R_{n}$ by a bounded (and relatively small) amount. If $P(A)$ is an ideal feature and all $R_{i} \in \overline{\bar{K}}$ satisfy $P(A)$ with a degree of satisfaction greater than zero and all $R_{j} \in \bar{C}$ fail to satisfy $P(A)$ (i.e., have degree of satisfaction with respect to $P(A)$ of less than zero), then $U$ may be mapped into a space $S$ whose size (number of elements) is a function of the difference of such degrees of satisfaction. No loss of recognition ability results while the consequent economy in computation is evident.

Also, a hierarchical theory of levels is utilized so that the problem of generating an ideal feature can be solved by stages, wherein at each except the final level, features which reduce the magnitude of the problem are generated. Here the "points" at each successive level represent the sets which satisfy the features at the previous level and the "atomic predicates" at each succes sive level derive from the features at the previous 
level. The method automatically advances one level whenever the number of elements in the regions to be partitioned (or the number of such regions) is thereby reduced. The procedure at each level is identical. A different "representation of the problem" is thereby expressed at each successive level (Nilssen, 1971; Amarel, 1962; Sherman and Ernst; 1969 ).

The material in this paper is organized as follows. The syntactic methods are described in the first portion. Features are defined as formulas in the weak second order predicate calculus with one free set variable. The atomic predicates used specify the positions of, darkness of, and other measurements at, and relations between, each of a set of data points called the data space.

These atomic predicates are called data independent if they do not vary for different inputs (e.g., the relative positions of the data points at which a photograph is sampled by a flying spot scanner are data independent, i.e., data independent predicates do not depend upon darkness, color, etc.). Atomic predicates are called data dependent if and only if they vary for different inputs (e.g., the darkness of a given data point on a particular photograph). Set membership is, of course, also a primitive of the system. Objects are regions (subsets) of the data space which satisfy a feature and which are maximal (i.e., they have no proper supersets which also satisfy the feature-this is called strongly satisfying the feature). A descriptive basis consists of all features generated by the learning algorithm at a particular stage in the operation of the system. A description consists of the descriptive basis and a list of the elements in each of the objects.

All formulae are put in distributive form. In such formulae the scope of each quantifier is as small as possible, negations occur only before atomic formulae, all subformulae within the scope of a universal quantifier are connected by disjunctions, and subformulae within the scope of an existential quantifier are connected by conjunctions. Such a formula is conveniently represented by a directed graph which is a rooted tree wherein the subtree below each node represents a subformula consisting of a quantifier (if such is present) and all subformulae within its scope. Each branch from that node represents a subformula within the scope of that quantifier, and unquantified atomic formulae are the bottom nodes of the tree.

All features have corresponding computer programs which evaluate that formula in a given space. If a formula is placed in prenex form and adjacent like quantifiers exchanged to generate additional prenex forms, distinct distributive forms may be obtained by operating on each such prenex form. This set of distributive forms is called the distributive set of the formula and each such distributive form corresponds to a computer program which can evaluate the formula.

A procedure called abstraction compares the distributive set of one feature with that of another to determine common subformulae (i. e. , common subprograms). Such sub- 
formulae are extracted, are called derived predicates and are retained by the system. In effect, abstraction extracts identical subtrees from the graphs of distinct features.

Another procedure called relation abstraction exposes the similarity of structure of pairs of trees with different atomic formulae. That is, formulae which differ only in that they contain different subformulae which, nonetheless, have the same free variables in corresponding positions, are extracted and retained. In these retained formulae, called predicate forms, differing subformulae in the features originally compared correspond to a dummy predicate, wherein the free variables are shown, but the identity of the predicate is not. Such dummy predicates will later be replaced by a derived predicate with the same free variables.

Derived predicates and predicate forms are compared to produce two hierarchies of formulae, the predicate hierarchy and the relational hierarchy, later used in the "learning algorithm".

Part 12 discusses relations between the syntax of the formulae and certain properties of those regions (i.e., subsets) of the data space which satisfy the formulae. Such regions are called models of the formula they satisfy and are compared both with those given regions we desire to be models of the formula, $\bar{K}=R_{1}, R_{2}, \ldots, R_{N}$, and those given regions we desire not to be models of the formula, $\bar{C}=R_{N+1}, \ldots, R_{N+M^{*}}$ Measures of the "similarity" between the actual models of a formula and the given regions determine various measures of success of the features generated. The ease of satisfaction of a formula is defined as the probability that a randomly selected element from the product space over which its free variables range satisfies the formula. The overlap of a pair of formulae is the ease of satisfaction of the conjunction of two formulae. Mutations map from one feature to another. Relaxing mutations take a formula into another which is implied by it (ease of satisfaction is increased), restricting mutations do the reverse and neutral mutations connect formulae which are not related by implication. Restricting and relaxing mutations (with the exception of quantifier exchanges) correspond to the addition or elimination of subtrees from some node on the graph of the formula, whereas neutral mutations correspond to replacements of subtrees of the graph, All are related to the abstraction procedure.

Each feature, $P_{i}$, is broken into a conjunction of two formulae, $P_{i}^{I}$ and $P_{i}^{D}$ wherein $\mathrm{P}_{i}^{\mathrm{I}}$ contains only data independent atomic predicates. We now examine the models of $\mathrm{P}_{i}^{\mathrm{I}}$ in two sets of spaces, one consisting of each of the given regions in $\overline{\mathrm{K}}$ and $\overline{\mathrm{C}}$ (some of which we wish to be models of $P_{i}$ and some which we do not) and the other consisting of each model of $\mathrm{P}_{\mathbf{i}}^{\mathrm{D}}$ (in the data space). Because models of a feature must strongly satisfy it, we do not, in this case, have the law of the excluded middle (i.e., a region which is not a model of $\mathbf{P}_{\mathbf{i}}$ need not be a model of $\sim p_{i}$ ), and we are able to derive results relating the ease of satisfac- 
tion of a given feature with that of an ideal feature which we are attempting to generate (i.e. , one of which each element of $\overline{\mathrm{K}}$ is a model and none in $\overline{\mathrm{C}}$ is). That is, by examining the size and number of models of a feature in the above spaces we are able to determine what kind of mutation is required.

One result of the above is: if a given region $R_{i}$ is an element of $\bar{K}$ (those we wish to be models of $P_{i}$ ), then (a) when $R_{i}$ does not satisfy $P_{i}$ a relaxing mutation on $P_{i}$ is required, and (b) when $R_{i}$ satisfies $P_{i}$ but not strongly, a restricting mutation is required. When $R_{i}$ is an element of $\bar{C}$ (those regions which must not be models of $P_{i}$ ) and $R_{i}$ strongly satisfies $P_{\dot{1}}$, any mutation may suffice (although a restricting mutation is usually preferred). These criteria are, of course, averaged for all elements of $\overline{\mathrm{K}}$ and $\overline{\mathrm{C}}$.

Now a more powerful technique of mutation selection is exposed: Suppose $R_{\hat{I}} \in \bar{K}$ and $R_{i}$ does not satisfy $P_{i}$. In $P_{i}$ each of the nested universal quantifiers (" $\left.\forall x^{\prime \prime}\right)$ is replaced one at a time, starting with the outermost (highest on the tree) by the modified quantifier " $\bar{Q}(\mathrm{k}) \mathrm{X}$ " which computes the fraction of substitutions of $\mathrm{x}$ (as constrained by any set membership atomic predicates, " $\in(x, A)$ " within the scope of that quantifier) for which the entire formula holds true (such fractions may equal zero). That is, the original universal quantifier, " $\forall x "$, is weakened to mean "for $k \cdot 100$ percent of $x$ " instead of "for all $x "$. This is done independently for each universal quantifier, and such a fractional value is thereby computed for each nested subformula within the scope of each universal quantifier (i.e., subtree on the graph). Clearly this value indicates the amount by which the "ease of satisfaction" of the subformula must be increased if the given set is to be a model of the resulting (modified) feature. The "degree of undersatisfaction" of a feature with respect to a given set is defined as the largest of the above computed fractional values minus one. After this procedure is completed, each of the existential quantifiers (" $\left.\exists x^{\prime \prime}\right)$ are, one at a time, replaced by "Q $(\mathrm{Q}) \mathrm{x}$ " and again a fractional value is computed for each nested subformula within the scope of an existential quantifier. Now each (except the bottom) node on the tree representing a formula has such a fractional value associated with it.

Now suppose $R_{i} \in \bar{K}$ and satisfies $P_{i}$ but not strongly. Only the latter of the above procedures is utilized: each existential quantifier is replaced by " $\bar{Q}(\mathrm{k}) \mathrm{x}^{\prime \prime}$ and a set of corresponding values is determined. The smallest of these fractional values is defined as the "degree of oversatisfaction" of the feature with respect to the given set.

It is then shown that modified calculations of the above fractional values can be used to estimate the ease of satisfaction of the various subformulae of a feature when that feature is evaluated. Thus, since derived predicates are extracted from features, their ease of satisfaction is automatically estimated and stored. These derived predicates are the subtrees which are added and eliminated from the graph of a feature when mutation is performed. Note that the replacement of universal quantifiers by a " $\bar{Q}(k) x^{\prime \prime}$ quantifier is equi- 
valent to a relaxing mutation of the formula consisting of the universal quantifier and the subformulae within its scope. Such an alteration of the quantifier is then repaired by performing an actual relaxing mutation on the subformula and restoring the universal quantifier. This mutation usually involves the addition or elimination of a subformula (i.e., derived predicate) whose choice is determined by its ease of satisfaction, which should be as consistent as possible with the fractional value of the modified quantifier. Neutral mutations can be similarly (but more weakly) determined by the estimated "overlap" (defined previously) of the required mutations.

The above procedures will not always uniquely select a mutation. When a feature is generated its measures of success are evaluated and a corresponding number called a reinforcement, which is assigned to that feature together with all derived predicates and predicate forms contained within it, is appropriately altered. Thus all formulae and predicate forms have such corresponding indications of their "success" thus far. When several mutations of a feature are equally appropriate, a selection is made as follows: The feature is compared with each formula in the list of predicate forms. That predicate form with the highest reinforcement which will result from a mutation of the required type and amount (of change) is chosen. The dummy predicates are either replaced by subformulae of the features being mutated, or if not therein present, such replacements are chosen from the list of derived predicates (with the required numbers of free point and set variables). The derived predicates with the highest reinforcements are chosen first. Hence the enormous possibilities of mutation choice are reduced first, by the semantic criteria resulting from examination of the models of the feature being altered, and second, by the syntactic criteria which favor those predicate forms and derived predicates which characterize previously successful features.

The next part of the paper deals with the technique for reducing the size of the problem by means of the use of a hierarchical level theory (discussed earlier) for representing the given data by the formulae in the descriptive basis. That is, a technique is given for automatically representing objects as collections of subobjects which are collections of subsubobjects, etc., all such objects being defined by features which are similarly hierarchically organized.

The following part of the paper treats the topology of the data space as related to techniques for computation reduction. The most important notion is a set of reduction mappings which successively reduce the size of the data space $(\mathrm{e} . \mathrm{g}$. , the fineness of grain of sampling). These mappings are related to invariances with respect to transformations which dilate or contract the space. The way in which resulting errors depend upon such a reduction mapping together with a particular feature are studied. 
The final part describes the overall systematic procedure for solving the problem. Various important open questions, whose solution would vastly affect the power of the system are discussed. For an illustrative example which simulates the procedures described, see Rothenberg (1973b).

It is important to note that the techniques developed permit a human trainer to simply and directly insert any knowledge or intuitions he has about the problem at any time during or prior to computation. Hence the task of problem solution begins at the limits of the trainer's knowledge and is less difficult than would otherwise be expected.

The description below is geared for pictorial pattern interpretation. It is easily seen, however, that other pattern interpretation problems fit the same formalism

\section{Syntax}

The sensory data or input consists of a digitized sampling of a (single) picture. This sampling is at a finite ordered array of points called the data space, $U=\left\{x_{1}, x_{2}, \ldots, x_{n}\right\}$, which is the same for all pictures. For each such data point (which corres ponds to a receptor in the sensory field such as a photosensitive cell), there is a vector of $\mathrm{k}$ values called its value vector, each element of which is called a data value. For illustrative purpos es we will here assume that the first two positions in the vector specify the row and column positions, respectively, (in the ordered array) of the data points. The remainder of the data values specify different quantized qualities (i.e., color or darkness) of that data point on a particular picture (these correspond to a quantized energy density at a particular receptor). The array of all value vectors (at all data points) is called the value matrix and is denoted $\left\|_{v}{ }^{(k)}\right\|$ where $k$ indexes the positions in the value vector the particular fineness of grain of sampling is specified by the size of the matrix). For convenience, each lower case letter, " $\mathrm{x}$ ", " $\mathrm{y}$ ", etc. will be used to designate a data point (i.e. the first two positions of the value vector), the $k^{\text {th }}$ data value of point $x$ will be denoted " $v "(k)(x)^{s t}$, and upper case letters, "A", " $\mathrm{B}^{\prime \prime}$, etc. will denote sets of data points.

Data values determine the truth values of a set of primitives (also called atomic predicates $1, \alpha^{(k, i)}(x)$, which are "true" if $v^{(k)}(x)=i$ and "false" otherwise. Those for which $k>2$ are called data dependent primitives because they differ for different input photographs.

Also given are a set of $n$ place primitives, $\beta\left(x_{1}, x_{2}, \ldots, x_{n}\right)$ which specify (geometrical) relations between data points. A typical example would be " $<(x, y, z, w) "$ which here

\footnotetext{
1 Conventionally, the term "primitive" refers to a specified "property" or relation (e.g. , " $\epsilon$ " for "set membership"), whereas "atomic predicate" refers to the primitive together with a specific s et of arguments (e.g. " " $\epsilon(\mathrm{x}, \mathrm{A}) "$ "). The usage of these terms here ignores the distinction.
} 
means that data point $\mathrm{x}$ is closer to $\mathrm{y}$ than $\mathrm{z}$ is to $\mathrm{w}$. This is usually derived from a given metric on the data space (which is a function of $v^{(1)}(x)$ and $v^{(2)}(x)$ - the row and column positions of point $x$ ). Such atomic predicates (as well as $\alpha^{(1, i)}(x)$ and $\left.\alpha^{(2, i)}(x)\right)$ are called data independent because they are the same for all input photographs.

The following definitions clarify the syntax used.

(1) Region: Any subset of a data space.

(2) Feature: A formula, P(A), in the second order predicate calculus with equality such that:

(a) point variables $(x, y, \ldots)$ range over data points, set variables $(A, B, \ldots)$ range over regions, and all constants $\left(c_{1}, c_{2}, \ldots\right)$ are data points, or sets of data points $\left(\mathrm{C}_{1}, \mathrm{C}_{2}, \ldots\right)$.

(b) all variables are bound except one set variable, A thence only regions can satisfy a feature).

(c) primitives include " $\epsilon(\mathrm{x}, \mathrm{A})$ " (conventionally " $\mathrm{x} \in \mathrm{A} ")$ and $"=(\mathrm{x}, \mathrm{y})$ " (conventionally $\left." \mathrm{x}=\mathrm{y}^{\prime \prime}\right)^{1}$, both of which are called elementary primitives. "card(A) < card(B)" may also be used.

(Also included are the data independent and dependent primitives (atomic predicates) defined above.)

Certain other conditions may be included which guard against formulae with trivial interpretations in the pictorial case. These will not be discussed here (s ee Rothenberg, 1973b).

(3) Object: A region which satisfies a feature (i. e., is defined as a realization of that feature) and is not a proper subset of any other realization of that same feature (i.e., it is "maximal). Note that while a region may be an object because strongly satisfies one feature, it may satisfy another feature, but not strongly. When an object satisfies a feature and is maximal with respect to that particular feature it will be said to strongly satisfy that feature and is called a model of the feature (as distinct from a realization). When a set of points satisfies a feature but is not maximal for that feature, it will be said to satisfy that feature, but not strongly.

(4) Descriptive Basis: All features which have been generated by the learning algorithm (which generates such features) at a particular stage in the adaptive development of the analysis.

$\overline{1}$ Note that although $"=(x, y)^{\prime \prime}$ will be used as an atomic predicate, it can, in the case of our illustrations, be defined as follows:

$=(x, y) \equiv \forall z\left(\sim<(x, z, y, z)_{\Lambda} \sim<(y, z, x, z)\right)$ (see ${ }^{n}=(x, y, z, w)^{n}$ definition in Example 1). 
(5) Description: This contains all features in the descriptive basis and lists all objects strongly satisfying each of these features and the elements of each such object. The satisfaction of a feature by an object which does not strongly satisfy that particular feature is also indicated.

(6) Object Class: A set of objects all of which strongly satisfy the same feature (also defined as the extension of the formula for that feature).

(7) Derived Predicates: A subformula of a feature which is recursively built up from primitives by the use of logical connectives and quantifiers. They are denoted " $D_{i}\left(x_{1}, \ldots, x_{n}, A_{1}, \ldots, A_{m}\right)$ " where $i$ indexes the predicate and its argument includes all free variables.

(8) Data Dependent Predicates: All formulae (including features) which contain data dependent primitives. All other formulae are called data independent.

\section{Example 1}

Let the primitives be " $\epsilon(X, A) ", "=(x, y) n$, and $"<(x, y, z, w) "$, which specifies that the distance between data point $\mathrm{x}$ and data point $\mathrm{y}$ is less than the distance between $\mathrm{z}$ and $w$. Also let " $\bar{D}(x)^{t}$ indicate that a data value (the third position in the value vector at data point $\mathrm{x}$ ) is equal to 1. Here we assume that these data values are either one or zero, the former indicating a "dark" and the latter a "light" point. Then examples of derived predicates are: ${ }^{1}$

$$
\begin{aligned}
& =(x, y, z, w) \equiv \sim<(x, y, z, w) \wedge \sim<(z, w, x, y) \quad\left("(x, y) \text { equals }(z, w)^{\prime \prime}\right)^{2} \\
& \mathrm{D}_{\mathrm{b}}(\mathrm{x}, \mathrm{y}, \mathrm{z}) \equiv \sim=[\mathrm{x}, \mathrm{y}] \wedge \sim=[\mathrm{x}, \mathrm{z}] \wedge \sim=[\mathrm{y}, \mathrm{z}] \wedge \vee \mathrm{W}(=[\mathrm{y}, \mathrm{w}] \vee<[\mathrm{x}, \mathrm{y}, \mathrm{x}, \mathrm{w}] \vee<[\mathrm{z}, \mathrm{y}, \mathrm{z}, \mathrm{w}]) \\
& (y \text { is "between" } x \text { and } z \text { ) } \\
& D_{c}(A, B) \equiv \forall x(\epsilon[x, A] \rightarrow \epsilon[x, B]) \quad\left(" A \text { is contained in } B^{\prime \prime}\right) \\
& \left.D_{s}(A, B) \equiv D_{c}(A, B) \wedge D_{c}(B, A) \quad \text { ("A is the same as } B "\right) \\
& \mathrm{D}_{\phi}(\mathrm{A}) \equiv \sim \exists \mathrm{x}(\epsilon[\mathrm{x}, \mathrm{A}]) \quad \text { ("A is the null set") } \\
& \mathrm{D}_{\mathrm{d}}(\mathrm{A})=\forall \mathrm{x}(\epsilon[\mathrm{x}, \mathrm{A}] \rightarrow \overline{\mathrm{D}}[\mathrm{x}]) \quad \text { ("all points in } \mathrm{A} \text { are 'dark' points") } \\
& \mathrm{D}_{\mathrm{a}}(\mathrm{x}, \mathrm{y})=\sim=[\mathrm{x}, \mathrm{y}] \wedge \nabla \mathrm{z}\left(\sim \mathrm{D}_{\mathrm{b}}[\mathrm{x}, \mathrm{z}, \mathrm{y}]\right) \quad\left(\text { (x is adjacent to } \mathrm{y}^{\prime \prime}\right)^{3}
\end{aligned}
$$

1 Note that throughout this description square brackets "[ $]$ " and round brackets " 0 " are used interchangeably and are alternated for clarity only.

2 Note that $"="$ when here used as a four place predicate, $"=(x, y, z, w) "$, has a different meaning than when used as a two place primitive, $n=(\mathrm{x}, \mathrm{y}) "$ (meaning that $\mathrm{x}$ and $\mathrm{y}$ are the same point).

3 For use with a coarse (sampling) grid it is suggested that $D_{a}(x, y)=\sim=(x, y) \wedge \forall z$ $(=[x, z] \vee \sim<[x, z, x, y]$ ) ("There is no point closer to $x$ than $y ")$ be used instead of the above. 
Note that all $y$ which satisfy $D_{b}(x, y, z)$ when $x$ and $z$ are fixed will approximate a "straight line" even if the grain of the data space is coarse. As the grain becomes infinitely fine these points approximate a "true" straight line.

For convenience ${ }^{1}$ we will use the symbol " $\psi\left(x_{1}, x_{2}, \ldots, x_{n}\right)$ " to denote that all variables within brackets denote distinct objects:

$$
\psi(x, y, z)=\sim=(x, y) \wedge \sim=(x, z) \wedge \sim=(y, z)
$$

Hence $D_{b}$ ("betweenness") in Example 1 may be rewritten

$$
\mathrm{D}_{\mathrm{b}}(\mathrm{x}, \mathrm{y}, \mathrm{z})=\psi(\mathrm{x}, \mathrm{y}, \mathrm{z}) \wedge \forall w(=[\mathrm{y}, \mathrm{w}] \vee<[\mathrm{x}, \mathrm{y}, \mathrm{x}, \mathrm{w}] \vee<[\mathrm{z}, \mathrm{y}, \mathrm{z}, \mathrm{w}]) \text {. }
$$

Note that a large number of formulae are tautologous unless their free variables are distinct.

\section{Semantics (Illustrations)}

The translation from formulae to programs is simple. Quantifiers become "do loops" whose ranges are determined by the membership predicates, e.g.

$$
\begin{aligned}
& \forall x_{1}\left[\sim \epsilon\left(x_{1}, A\right) \vee P\left(x_{1}, \ldots, x_{n}\right)\right] \equiv \underset{x_{1} \in A}{\wedge} P\left(x_{1}, \ldots, x_{n}\right) \\
& \exists x_{1}\left[P\left(x_{1}, \ldots, x_{n}\right)\right] \equiv \underset{x_{1} \in U}{\vee} P\left(x_{1}, \ldots, x_{n}\right) \\
& \exists x_{1}\left[\epsilon\left(x_{1}, A\right) \wedge P\left(x_{1}, \ldots, x_{n}\right)\right] \equiv \underset{x_{1} \in A}{\vee} P\left(x_{1}, \ldots, x_{n}\right)
\end{aligned}
$$

("U" is the universal set - i.e., the data space) ${ }^{2}$

Logical connectives are functions computed by subroutines, and atomic and derived predicates become subroutines in the main program expressing the feature.

Some examples of the richness of the system when the primitives of Example 1 are used are:

$P_{1}(A)=\forall x \forall y \forall z\left(\epsilon[x, A] \wedge \in[z, A] \wedge D_{b}[x, y, z] \rightarrow \epsilon[y, A]\right)$ is data independent and satisfied by convex sets only $\left(D_{b}[x, y, z]\right.$ is defined as in Example 1$)$. If $P_{1}(A)$ is a feature, then, by definition, only maximal regions are objects.

$$
\text { Let } D_{d}(A) \equiv \forall x(\epsilon[x, A] \rightarrow \bar{D}[x]) \text { (as before) . }
$$

Then

$$
P_{2}(A) \equiv D_{d}(A) \wedge P_{1}(A)
$$

is a data dependent version of $\mathrm{P}_{1}$ (A)

\footnotetext{
1 Although not described her"e, the language has been generalized to include "syntactic variables" which range over formulae and primitives which specify whether these variables are bound or free. Programming is thereby facilitated when the language is used as a compiler (see Pessin and Rothenberg reference).

2 Quantified set variables range over regions. Methods of reducing such computation are discussed in Rothenberg (1973b)
} 


$$
P_{3}(A) \equiv \exists x\left[\epsilon(x, A) \wedge \forall y \forall z\left(\epsilon[y, A] \wedge D_{b}[x, z, y] \rightarrow \epsilon[z, A]\right)\right]
$$

defines a "starlike" region, and a derived predicate which is satisfied only by a boundary point, $x$, of a region, $A$, is given by

$$
D_{1}(x, A) \equiv \epsilon(x, A) \wedge \exists y\left(\sim \in[y, A] \wedge D_{a}[x, y]\right)
$$

A feature satisfied only by a connected ${ }^{1}$ region can be constructed in two steps:

$$
\mathrm{D}_{2}(\mathrm{~A}, \mathrm{~B}) \equiv \forall \mathrm{xVy}\left(\epsilon[\mathrm{x}, \mathrm{A}] \wedge \in[\mathrm{y}, \mathrm{B}] \wedge \mathrm{D}_{\mathrm{a}}[\mathrm{x}, \mathrm{y}] \rightarrow \epsilon[\mathrm{y}, \mathrm{A}]\right)
$$

is a derived predicate which asserts that $\mathrm{A}$ is "closed" in $\mathrm{B}$ under the relation of adjacency and

$$
P_{6}(A) \equiv V B\left(D_{c}[B, A] \wedge \sim D_{\phi}[B] \wedge D_{2}[B, A] \rightarrow D_{s}[B, A]\right)
$$

is satisfied iff $A$ is a connected region ("for all $B$, if $B \subset A$ and $B \neq \phi$ and $D_{2}[B, A]$ then $B=A^{\prime \prime}$-see Example 1). Other equivalent formulae exist.

Simple connectedness ("A has no holes") is easily expressed by stating that the boundaries of both $\mathrm{A}$ and its complement are connected sets. Other illustrations are easily constructed.

An example of a data dependent feature is $P_{7}(A)$ which accommodates "noisy" dark areas:

$$
P_{7}(A) \equiv \forall x\left(\epsilon[x, A] \rightarrow \bar{D}(x) \vee \forall y\left[D_{a}(x, y) \rightarrow \bar{D}(y)\right]\right)
$$

\section{Distributive Normal Forms}

This is a form in which all quantifiers are distributed as much as possible along the terms of a formula so that a minimum number of terms lie within the scope of each quantifier. (It is the opposite of a prenex normal form and is similar to, but not identical to, the "mini-scope" of Wang (1960) and somewhat resembles the "distributive normal forms" of Hintikka (1953). This is accomplished on a formula by reversing the techniques for placing formulae in prenex normal form (Rothenberg, 1971). A formula is in distributive normal (henceforth abbreviated "distributive") form iff:

(1) Negations appear only before atomic subformulae.

(2) All subformulae ${ }^{2}$ within the scope of a universal quantifier are connected by disjunctions.

1 By "connected region" is intuitively meant that for each pair of sub-regions which partition the region there exists a pair of adjacent points, one of which is contained in each subregion.

2 A subformula is either an atomic predicate or derived predicate or either of these quantified and/or negated. 
(3) All subformulae within the scope of an existential quantifier are connected by conjunctions.

(4) All subformulae within the scope of a quantifier are either prefixed by a quantifier or are atomic formulae or their negations. (That is, they are not bracketenclosed conjunctions or disjunctions of other subformulae.)

(5) All subformulae within the scope of a quantifier contain the quantified variable as an argument.

(6) If two or more like quantifiers are adjacent (e.g., " $\nabla x \forall y "$ or " $\exists x \exists y ")$, all subformulae within their scope must contain such adjacently quantified variables as arguments.

\section{Example 2.}

Consider the formula

$$
\mathrm{P}_{8}(\mathrm{~A}) \equiv \operatorname{Vx} \exists \mathrm{y}\left([\epsilon(\mathrm{y}, \mathrm{A}) \vee \epsilon(\mathrm{x}, \mathrm{A})] \rightarrow \exists \mathrm{z}\left[\mathrm{D}_{\mathrm{b}}(\mathrm{x}, \mathrm{y}, \mathrm{z}) \wedge \in(\mathrm{z}, \mathrm{A})\right]\right) \text {. }
$$

It has only one distributive form (in general, this is not the case-to be discussed):

$$
\begin{aligned}
& P_{8}(A) \equiv \forall x(\sim \epsilon[x, A]\left.\vee \exists\left[(z, A) \wedge \exists y\left(D_{b}[x, y, z]\right)\right]\right) \\
& \wedge \exists y(\sim \in[y, A]) \vee \forall x \exists z\left(\epsilon[z, A] \wedge \exists y\left(D_{b}[x, y, z]\right)\right)
\end{aligned}
$$

For convenience we here insist that, within the scope of each quantifier, (a) set membership primitives precede all others, (b) quantified terms be placed last, and (c) those terms whose arguments contain fewer variables precede terms whose arguments contain a greater number of variables.

Note that, because of (1) - (6) above, a distributive formula may be easily represented by directed graph which is a rooted tree wherein the subtree below each node represents a subformula consisting of a quantifier (if such is present) and all subformulae within its scope. ${ }^{1}$ Each branch from that node represents a subformula within the scope of that quantifier, and unquantified atomic formulae (and their negations) are the nodes with no successors. The number of distinct bound variables in the formula corres ponds to the number of edges in the longest path down the tree. Circular nodes will be used to contain quantifiers of point variables, two concentric circles will contain quantifiers of $s$ et variables, and rectangular nodes will contain atomic predicates. The point variables will be indexed in their order of quantification (starting at the top of the tree) by integers. Set variables will be similarly indexed by Roman numerals. Free point variables will be denoted by

\footnotetext{
1 When the entire formula is not quantified but is instead a logical combination of formulae which are quantified, the top nodes of the graph correspond to connectives " $\wedge$ " or " $\vee$ " instead of quantifiers. The graph representation is here used only for illustrative purposes.
} 
$0_{1}, 0_{2}, \ldots$ etc. in the order in which they appear in atomic predicates. Free set variables will be similarly denoted $\phi_{1}, \phi_{2}, \ldots$ etc. When a feature is represented, there will be only one free variable, the set variable, $\phi$.

Here an example of a first order feature is shown (for a graph of a second order feature see Example 5):

\section{Example 3}

Consider the feature $P_{10}(A)$, which is satisfied by regions which are "straight lines" containing more than two points: A distributive normal form of this formula is

$$
\begin{aligned}
P_{10}(A) \equiv & \exists x[\epsilon(x, A) \wedge \exists z(\epsilon[z, A] \wedge \forall y[\sim \in(y, A) \vee \psi(x, y, z)] \wedge \\
& \forall y[\sim \epsilon(y, A) \vee \forall w(=[y, w] \vee<[x, y, x, w] \vee<[z, y, z, w])])] .
\end{aligned}
$$

A graph corresponding to this formula is

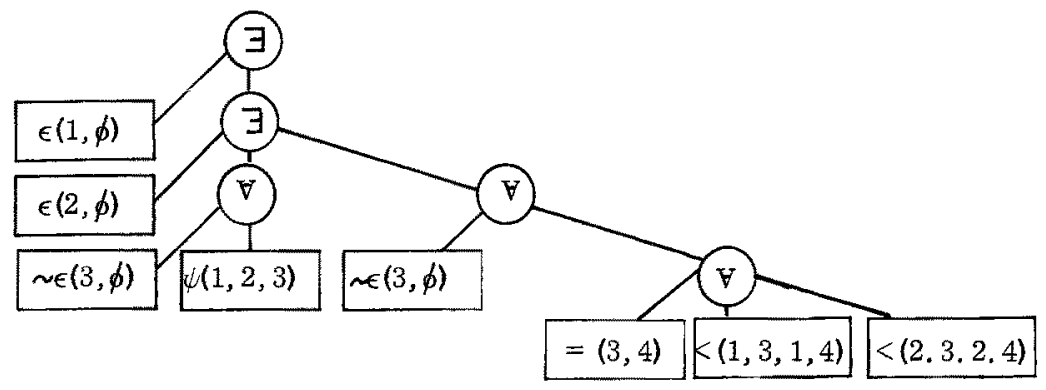

Thus, to decode the above graph, we may begin at the bottom quantifier: Let $A=\phi, x=x_{1}$, $y=x_{2}, z=x_{3}, w=x_{4}$ and we obtain the following subformula which we will denote $" \mathrm{D}_{1}(\mathrm{x}, \mathrm{y}, \mathrm{z}, \mathrm{A}) "($ where $\mathrm{x}, \mathrm{y}, \mathrm{z}$ and $\mathrm{A}$ are the free variables):

$$
\forall w(=[z, w] V<(x, z, x, w] V<(y, z, y, w]) \equiv D_{1}(x, y, z, A)
$$

If we include the note above it we obtain

$$
\forall z\left(\sim \in[z, A] \vee D_{1}[x, y, z, A]\right) \equiv D_{2}(x, y, A)
$$

Now if we write the formula corresponding to the node to the left of the one just considered:

$$
\forall z(\sim \in[z, A] \vee \psi[x, y, z]) \equiv D_{3}(x, y, A)
$$

Moving up a node on the graph we obtain:

$$
\exists y\left(\epsilon[y, A] \wedge D_{2}[x, y, A] \wedge D_{3}[x, y, A]\right) \equiv D_{4}(x, A) .
$$

Proceeding to the top node we obtain 


$$
\exists \mathrm{x}\left(\epsilon[\mathrm{x}, \mathrm{A}] \wedge \mathrm{D}_{4}[\mathrm{X}, \mathrm{A}]\right) \equiv \mathrm{P}_{10}(\mathrm{~A})
$$

which is an alphabetic variant of the formula for $P_{10}(A)$ given at the beginning of the example.

Note that this graph representation ensures that the minimum number of variables is used (Rothenberg, 1973b). In general, prenex normal formulae have more than one distributive form; also, a given distributive formula will often have more than one prenex form (e.g., when two branches from the same node contain differing quantifiers and all branches from that node contain quantifiers, e.g., $\forall x(\forall y[p(x, y)] \vee \exists y[q(x, y)])$ can be put into the prenex normal forms $\forall x \forall y \exists z(p[x, y] \vee q[x, z])$ and $\forall x \exists z \forall y(p[x, y] \vee q[x, z])$.)

Note that the prenex form has more quantified variables than the distributive form. This is often the case.

We are here interested in the distributive set of a formula. This is defined as the set of formulae obtained by placing a distributive formula in prenex form, obtaining all prenex forms resulting from the exchange of adjacent like quantifiers, placing each of such prenex forms in distributive form and eliminating the identical formulae which result (more economical methods exist).

Another form of interest here is called the nested form. This corresponds to a prenex formula wherein the quantifiers are imported ${ }^{1}$ into the subformulae within this scope until a further such importation would result in a circular node on the graph having two circular nodes as immediate succes sors. Such a formula is obtained from a distributive formula, whenever two or more quantified subformulae (i.e. subtrees on the graph) are the immediate success ors of a quantifier (circular node on graph), by consolidating such subformulae within the scope of a single quantifier. A nested form of the formula in Example 3 is (also see Example 5):

$$
\begin{aligned}
\mathrm{P}_{10}(A) \equiv & \exists \mathrm{x}[\epsilon(\mathrm{x}, \mathrm{A}) \wedge \exists \mathrm{z}(\epsilon[\mathrm{z}, \mathrm{A}] \wedge \forall \mathrm{y}[\sim \in(\mathrm{y}, \mathrm{A}) \\
& \vee(\psi[\mathrm{x}, \mathrm{y}, \mathrm{z}] \wedge \nabla \mathrm{w}[=(\mathrm{y}, \mathrm{w}) \vee<(\mathrm{x}, \mathrm{y}, \mathrm{x}, \mathrm{w}) \vee<(\mathrm{z}, \mathrm{y}, \mathrm{z}, \mathrm{w})])])] .
\end{aligned}
$$

Note that both conjunctions and disjunctions now appear within the scope of the quantifier of the variable, $y$. Nested formulae may be graphed by including nodes containing disjunctions and conjunctions. The graph of the nested form of $P_{10}(A)$ above is

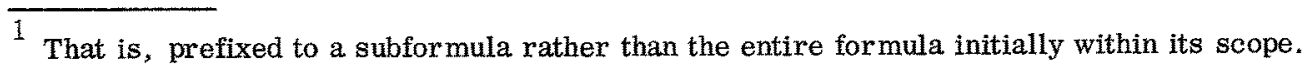




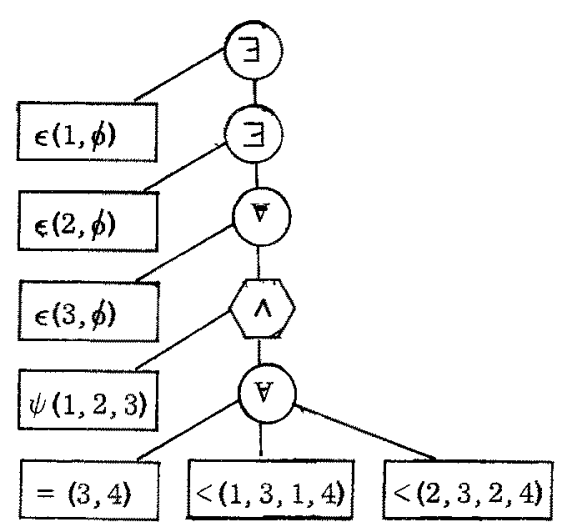

Although formulae, if in distributive normal form, translate into efficient computer programs (nested form is more efficient when it contains no more variables than the distributive form), the principal motivation here is the necessity for such form in the procedures which follow. Also, the "learning" algorithm generates and operates upon formulae in their distributive form.

\section{Compression and Decompression of Formulae}

In order to compare different formulae which contain identical derived predicates as subformulae, it is necessary to guarantee that such will not be concealed by their form. Hence, we define the following procedures:

Compression: This operation consists of replacing an innermost phrase of a formula by an abbreviation, $D_{i}\left(X_{1} \ldots x_{n}, A_{1} \ldots A_{m}\right)$, with the number of arguments equal to the number of free variables (see Example 3 above). If this predicate is a derived predicate which has already been generated (to be discussed), substitute its abbreviation. This is called one layer of compression. This may be repeated until all phrases are exhausted. When formulae for (all) existing derived predicates are replaced by their abbreviations, the resulting formula is defined as in collapsed form.

\section{Example 4}

$$
\begin{aligned}
& P_{1}(A) \equiv \forall x\left(\sim \epsilon[x, A] \vee \forall y[\epsilon(y, A) \vee \underbrace{\forall z\left(\sim \epsilon[X, A] \vee \sim D_{b}[X, y, z]\right.}]\right) \\
& P_{1}(A) \equiv \forall x(\sim \epsilon[x, A] \vee \underbrace{\forall y\left[\epsilon(y, A) \vee D_{1}(x, y, A]\right)}) \\
& P_{1}(A) \equiv \forall x\left(\sim \in[x, A] \vee D_{2}[x, A]\right)
\end{aligned}
$$

Decompression: This is the rever $\mathrm{s} e$ of compression where each derived predicate has its definition substituted for its abbrevation in the formula being decompressed. This is done one derived predicate at a time, working from the innermost outward. Each such 
substitution is defined as one layer of decompression. This may be repeated until only primitives remain, in which cas e the resulting formula is called the complete formula. In Example 3 above, we would substitute

$$
\sim \psi(\mathrm{x}, \mathrm{y}, \mathrm{z}) \vee \exists w(\psi[\mathrm{y}, \mathrm{w}] \wedge \sim<[\mathrm{x}, \mathrm{y}, \mathrm{x}, \mathrm{w}] \wedge \sim<[\mathrm{y}, \mathrm{z}, \mathrm{w}, \mathrm{z}]) \text { for } \sim \mathrm{D}_{\mathrm{b}}(\mathrm{x}, \mathrm{y}, \mathrm{z}) .
$$

\section{Simple Abstraction}

This is a procedure whereby the collapsed (distributive) forms in the distributive sets of $P_{i}$ and $P_{j}$ (or the graph of these forms) are mechanically compared and common subformulae of maximum length (i.e., with maximum number of contiguous symbols) are extracted and form derived predicates. Each comparison proceeds from terms of the innermost phrase outward to the remaining phrases. ${ }^{1}$ Whichever of $P_{i}$ or $P_{j}$ has fewer distinct collapsed forms is chosen, say $P_{j}$. Each such form is compared with any collapsed form of $\mathrm{P}_{\mathbf{i}}$ and $\sim \mathrm{P}_{\mathbf{i}}$. The longest subformulae which are common to both formulae are extracted and labeled as derived predicates. Permutation of terms within a phrase in order to extract a longer formula is permitted. Also permitted are exchanges of variables in primitives where the extension remains unchanged thereby $(e . g .,<(x, y, z, w) \equiv<(y, x, z, w))$. Substitutions of equivalent primitives may also be used, e.g. , $\sim<(x, y, x, z)=<(x, z, x, y) \vee$ $=(x, y, x, z)$. When comparison is made, if a distributive (collapsed) form deviates from nested (collapsed) form because a quantifier has been distributed through brackets, an additional comparison is made after nested form has been restored (see Example 5 below).

Notice that the use of collapsed forms guards agains excessive computation by not manipulating formulae for existing derived predicates (which resulted from simple abstraction previously performed).

\section{Example 5}

Here we compare (the distributed form of) a formula, $P_{9}(A)$, which is strongly satisfied by the union of all boundary points of dark circular regions (i.e., those regions which satisfy derived predicate $D_{11}(A) \equiv \exists x \exists y \exists(\psi[x, y] \wedge[\epsilon(z, A) \longleftrightarrow<(x, z, x, y)])$ with feature, $\mathrm{P}_{10}(\mathrm{~A})$, of Example 4 (for convenience $\psi(\mathrm{x}, \mathrm{y}, \mathrm{z})$ and $\sim \psi(\mathrm{x}, \mathrm{y}, \mathrm{z})$ will not be decompressed):

$$
\begin{aligned}
P_{g}(A) \equiv & \forall x\left[\sim \epsilon ( x , A ) \vee \exists B \left(\epsilon[x, B] \wedge D_{11}[B] \wedge D_{d}[B] \wedge \exists y[\sim \epsilon(y, B) \wedge \psi(x, y)\right.\right. \\
& \wedge \forall z(\sim \psi[x, y, z] \vee \exists w[\psi(z, w) \wedge \sim<(x, z, x, w) \wedge \sim<(y, z, y, w)])])] \\
P_{10}(A) \equiv & \exists x[\epsilon(x, A) \wedge \exists z(\epsilon[z, A] \wedge \forall y[\sim \epsilon(y, A) \vee \psi(x, y, z)] \\
& \wedge \forall y[\sim \epsilon(y, A) \vee \forall w(=[y, w] \vee<[x, y, x, w] \vee<[z, y, z, w])])] .
\end{aligned}
$$

I That is, those strings of symbols (starting from the right of the distributive formulae) which are common to both formulae and which contain no proper substrings common to both are extracted. 
Although $\mathrm{P}_{10}$ (A) is in distributed form, it does not conform to nested form Hence we consolidate all terms quantified by "Vy" within the scope of a single such quantifier:

$$
\begin{aligned}
P_{10}(A) \equiv & \exists x[\epsilon(x, A) \wedge \exists z(\epsilon[z, A] \wedge \forall y[\sim \epsilon(y, A) \\
& \vee(\psi[x, y, z] \wedge \forall w[=(y, w) \vee<(x, y, x, w) \vee<(z, y, z, w)])])]
\end{aligned}
$$

$P_{9}(A)$ has another distributive form, but we here take the negation of the above form and lainel our variables so that their order in the innermost phrase is similar to the order of variables in the innermost phrase of $\mathrm{P}_{10}(\mathrm{~A})$ :

$$
\begin{aligned}
\sim P_{9}(A) \equiv & \exists x\left[\epsilon ( x , A ) \wedge \forall B \left(\sim \in[x, B] \vee \sim D_{11}[B] \vee D_{d}[B] \vee \forall z[E(z, B) \vee=(x, z)\right.\right. \\
& \vee \exists y(\psi[x, z, y] \wedge \forall w[=(y, w) \vee<(x, y, x, w) \vee<(z, y, z, w)])])]
\end{aligned}
$$

A graphical repres entation of $\sim \mathrm{P}_{9}(\mathrm{~A})$ is

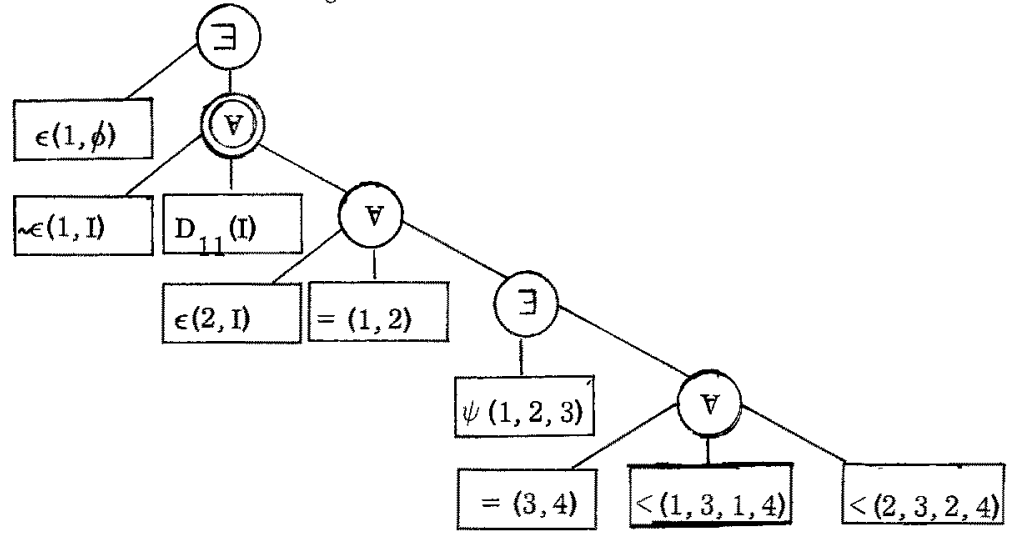

Comparison (from right to left) yields the common maximum subformula

$$
D_{b}(x, y, z)=\psi(x, y, z) \wedge \forall w[=(y, w) \vee<(x, y, x, w) \vee<(z, y, z, w)]
$$

and we see that

$$
\mathrm{P}_{10}(\mathrm{~A}) \equiv \exists \mathrm{x} \exists \mathrm{z}\left(\epsilon[\mathrm{x}, \mathrm{A}] \wedge \in[\mathrm{z}, \mathrm{A}] \wedge \forall \mathrm{y}\left[\epsilon(\mathrm{y}, \mathrm{A}) \rightarrow \mathrm{D}_{\mathrm{b}}(\mathrm{x}, \mathrm{y}, \mathrm{z})\right]\right)
$$

and if we substitute $D_{a}(x, y)$ for $\psi(x, y) \wedge \forall z\left(\sim D_{b}[x, z, y]\right)$ ("x adjacent to $y "$, see Example 1),

$$
p_{g}(A)=\forall x \exists B\left(\epsilon[x, A] \rightarrow \epsilon[x, B] \wedge D_{11}[B] \wedge D_{d}[B] \wedge \exists y\left[\sim \epsilon(y, B) \wedge D_{a}(x, y)\right]\right)
$$

This procedure is more easily seen by extracting common subtrees of the graphs of $\sim \mathrm{P}_{9}$ (A) and $P_{10}(A)$ (see previous section).

\footnotetext{
${ }^{1}$ Although not done in the diagrams above, for mechanized execution of the abstraction procedure, variables should be indexed in reverse order of quantification (i.e., starting from
the bottom of the tree).
} 
Comparison of even the simplest formulae often yields derived formulae. See Rothenberg (1973b) for examples.

In essence, the formulas obtained by abstraction (and by "relation abstraction", to be discussed) are those "properties" of features we consider in this system.

\section{The Predicate Hierarchy}

Complete abstraction is the application of the techniques of simple abstraction to complete formulae (i.e., formulae which have been decompressed till only primitives remain-see Section 5). This is performed at critical points in the flow of the system, such as when computer memory is becoming exhausted. Then all derived predicates retained by the system are eliminated and complete abstraction is performed with all pairs of features in the descriptive basis (i.e., "properties" of features are found). The derived predicates obtained are retained by the system (for purposes to be described in Section 15) and each pair of these is again compared to extract maximal common subformulae which again become derived predicates (i. e., "properties of properties" of features are found). This procedure continues untill only primitives remain, and the resulting set of derived predicates is called the predicate hierarchy. In this manner an economical representation of the structure of the descriptive basis may be obtained.

\section{Relation Abstraction}

Here we compare two formulae (again in compressed form), which although they possess no common subformulae, have identical logical form (i. e., identical relations between their subformulae), or which have subformulae of identical logical form. Consider

$$
P_{15}(A) \equiv \forall x \forall y\left(\epsilon[x, A] \wedge D_{a}[x, y] \rightarrow \epsilon[y, A]\right)
$$

which expresses closure under the relation of adjacency, and

$$
P_{16}(A)=\operatorname{Vx\forall y}\left(\epsilon[\mathrm{x}, \mathrm{A}] \wedge D_{5}[\mathrm{x}, \mathrm{y}] \rightarrow \epsilon[\mathrm{y}, \mathrm{A}]\right)
$$

which defines a region closed under another binary relation, $D_{5}(x, y)$. When relation abstraction is here performed a dummy predicate $K(x, y)$, replaces the differing binary predicates in the two formulae, and a predicate form results:

$$
\mathrm{S}(\mathrm{A}, \mathrm{K}[\mathrm{x}, \mathrm{y}])=\forall \mathrm{v} \forall \mathrm{y}(\epsilon[\mathrm{x}, \mathrm{A}] \wedge \mathrm{K}[\mathrm{x}, \mathrm{y}] \rightarrow \epsilon[\mathrm{y}, \mathrm{A}])
$$

This variable predicate expresses closure with respect to some unspecified binary relation $\mathrm{K}[\mathrm{x}, \mathrm{y}]$.

The procedure for comparing $P_{i}$ and $P_{j}$ is as follows: Compress whichever of $P_{i}$ and $P_{j}$ has the most variables until both formulae contain the same number of variables.

${ }^{1}$ See Example 4 for illustration of how the number of variables is reduced by compression. 
If the substitution of $k$ (at first $k=1$ ) distinct dummy predicates in each of $\mathrm{P}_{\mathbf{i}}$ and $\mathrm{P}_{\mathbf{j}}$ makes them identical, such resulting formula is a predicate form and is retained by the system. (This compression makes use of the negation of a formula and the other devices used in simple abstraction.) When such a substitution does not render $P_{i}$ and $P_{j}$ identical, both formulae are compressed one layer and the comparison procedure is repeated, ${ }^{1}$

Subformulae of a phrase may be permuted or combined, and a dummy predicate may be inserted where no subformula is present if needed to avoid combining two subformulae (one of which is in the other formula) into a single formula. (That is, if $P_{i}(x, y) \wedge P_{j}(y, z)$ $\wedge P_{k}(y, z, w)$ is part of a phrase being compared with $P_{i}(x, y) \wedge P_{k}(y, z, w)$, the latter becomes $P_{i}(x, y) \wedge K(y, z) \wedge P_{k}(y, z, w)$ and $K(y, z)$ is also substituted for $P_{j}(y, z)$ in the former.)

\section{Example 6}

Compare a predicate which is strongly satisfied by the boundaries of all dark convex $s$ ets

$$
\mathrm{P}_{17}(\mathrm{~A}) \equiv \forall \mathrm{Vx}\left(\epsilon(\mathrm{x}, \mathrm{A}) \rightarrow \exists \mathrm{B}\left[\epsilon(\mathrm{x}, \mathrm{B}) \wedge \mathrm{P}_{2}(\mathrm{~B}) \wedge \exists \mathrm{y}\left(\sim \epsilon(\mathrm{y}, \mathrm{B}) \wedge \mathrm{D}_{\mathrm{a}}(\mathrm{x}, \mathrm{y})\right)\right]\right)
$$

with a predicate which defines the boundaries of all dark sets with property $P_{3}(A)$ :

$$
P_{18}(A) \equiv \forall x\left(\epsilon(x, A) \rightarrow \exists B\left[\epsilon(x, B) \wedge \forall x(\epsilon(x, B) \rightarrow \bar{D}(x)) \wedge P_{3}(B) \wedge \exists y\left(\sim \epsilon(y, B) \wedge D_{a}(x, y)\right)\right]\right.
$$

Putting both in distributive normal form:

$$
\begin{gathered}
P_{17}(A) \equiv \forall x\left(\sim \epsilon(x, A) \vee \exists B\left[\epsilon(x, B) \wedge P_{2}(B) \wedge \exists y\left(\sim \epsilon(y, B) \wedge D_{a}(x, y)\right)\right]\right) \\
P_{18}(A) \equiv \forall x\left(\sim \epsilon ( x , A ) \vee \exists B \left[\epsilon(x, B) \wedge \forall z(\sim \epsilon(z, B) \vee \vec{D}(\mathrm{z})) \wedge P_{3}(B)\right.\right. \\
\left.\left.\wedge \exists y\left(\sim \in(y, B) \wedge D_{a}(x, y)\right)\right]\right)
\end{gathered}
$$

$P_{17}$ (A) has fewer variables than $P_{18}(A)$. Hence compression of $P_{18}(A)$ (indicated by bracket under above formula) yields:

$$
\mathrm{P}_{18}(\mathrm{~A}) \equiv \forall \mathrm{x}\left(\sim \epsilon(\mathrm{x}, \mathrm{A}) \vee \exists \mathrm{B}\left[\epsilon(\mathrm{x}, \mathrm{B}) \wedge \mathrm{D}_{1}(\mathrm{~B}) \wedge \mathrm{P}_{3}(\mathrm{~B}) \wedge \exists \mathrm{y}\left(\sim \epsilon(\mathrm{y}, \mathrm{B}) \wedge \mathrm{D}_{\mathrm{a}}(\mathrm{x}, \mathrm{y})\right)\right]\right)
$$

The next to innermost phrase above has a greater number of subformulae than the next to innermost phrase of $\mathrm{P}_{17}(\mathrm{~A})$. Hence subformulae of $\mathrm{P}_{18}(\mathrm{~A})$ are combined (as indicated by bracket under above formula) to yield:

$$
\mathbf{P}_{18}(\mathrm{~A}) \equiv \forall \mathrm{x}\left(\sim \epsilon(\mathrm{x}, \mathrm{A}) \vee \exists \mathrm{B}\left[\epsilon(\mathrm{x}, \mathrm{B}) \wedge \mathrm{D}_{2}(\mathrm{~B}) \wedge \exists \mathrm{y}\left(\sim \epsilon(\mathrm{y}, \mathrm{B}) \wedge \mathrm{D}_{\mathrm{a}}(\mathrm{x}, \mathrm{y})\right)\right]\right)
$$

$\overline{1}$ The entire procedure may be repeated for $k=1,2, \ldots, n$ ( $n$ is chosen to conform with the availability of computer memory and to avoid excessive iteration. Hence it will usually be small.) 
It is now easy to see that comparison of the compressed sets for $P_{17}(A)$ and $P_{18}(A)$ above will yield the following predicate form:

$$
\mathrm{s}_{2}(A, K(B)) \equiv \forall x\left(\sim \epsilon(x, A) \vee \exists B\left[\epsilon(x, B) \wedge K(B) \wedge \exists y\left(\sim \epsilon(y, B) \wedge D_{a}(x, y)\right)\right]\right)
$$

which defines the boundaries of all regions, $B$, which satisfy $K(B)$.

Again, the above procedure can be performed by comparison of the graphs of $P_{17}(A)$ and $P_{18}(A)$ for similarity of structure (exclusive of the different atomic formulae).

Relation abstraction is executed whenever simple abstraction is, and predicate forms are also retained by the system ${ }^{1}$ (for uses to be described in Section 15). When complete abstraction is performed and the predicate hierarchy is restructured, relation abstraction is also performed on complete formulae and, in similar fashion, a hierarchy of predicate forms (called the relational hierarchy) is constructed. ${ }^{2}$ At each successive level in this hierarchy the predicate forms acquire additional dummy variables. None are retained with more than $\mathrm{n}$ such dummy variables.

\section{An Elimination Procedure for the Set Membership Primitive}

For our purposes we define a feature, $P(A)$ as a tautology iff $P$ is satisfied by all subsets of the data space or only by the entire data space. $P(A)$ is defined as a contradiction iff $P$ is satisfied by no subset of the data space.

Now we eliminate vacant predicates and tautologies which derive from the use of the primitive, " $\in(x, A)$ ". Contradictions and tautologies are characterized by: $\forall A(P[A])$ and $\sim \exists_{A}(P[A])$. These formulae have a similar significance: (a) $\forall \mathrm{x}(\epsilon[\mathrm{x}, \mathrm{A}])$ and (b) $\sim \exists x(\epsilon[x, A])$ or $\forall x(\sim \epsilon[x, A])$. (We are not interested in the empty set or its complement.) Hence the following partial decision procedure: (1) Examine a formula and its negation (both in complete and distributive form). (2) If a term of form (a) (or (b)) occurs, label it "T" (or "F"). (3) If "T" (or "F") occurs as part of a conjunction or a quantified conjunction, label the conjunction "T" (or " $F "$ "). (4) If the entire formula becomes labelled "T" (or " $F "$ ), the formula is tautologous (or contradictory). The revers e is true for its negation. Hence,

$$
P_{19}(A) \equiv \forall x \forall y \exists z\left(\epsilon[x, A] \wedge \epsilon[y, A] \wedge D_{b}[x, z, y] \rightarrow \epsilon[z, A]\right)
$$

which has the following distributive form of its negation

$$
\sim P_{19}(A)=\exists x\left(\epsilon[x, A] \wedge \exists y\left[\epsilon(y, A) \wedge \forall z\left(D_{b}[x, z, y]\right) \wedge \forall z(\sim \epsilon[z, A])\right]\right)
$$

is tautologous (examine its last term) and will not be generated by the system.

\footnotetext{
1 All nested forms of a predicate form are retained by the system.

2 Actually, relation abstraction is also performed on derived predicates as well as features.
} 
Similar procedures can often be developed to eliminate tautologies and and contradictions resulting from the use of other primitives. (The above is, in essence, the familiar technique of "quantifier elimination".) This of course, depends upon the choice of primitives in the particular application.

Other peculiarities of the system permit the elimination of other useless formulae. Notice that because all objects are maximal regions, if

$$
\left[\exists x_{1}, \ldots, \exists x_{n} \forall y\left(\epsilon[y, A] \rightarrow D\left[x_{1}, \ldots, x_{n}, y\right]\right)\right]
$$

is a data independent feature, an object which strongly satisfies it also satisfies

$$
\left[\exists x_{1}, \ldots, \exists x_{n} \nabla y\left(D\left[x_{1}, \ldots, x_{n}, y\right] \rightarrow \epsilon[y, A]\right)\right]
$$

Notice that if a formula for a feature is used as part of another feature it becomes a derived predicate and the maximality condition no longer applies. In such case "↔" must be substituted for " $\longrightarrow$ " in the first of the above formulae if its meaning is to remain unchanged.

Consider a feature with a collapsed form

$$
P(A) \equiv \forall x\left[\epsilon(x, A) \vee D_{F}(x)\right] \equiv \forall x\left[\sim \epsilon(x, A) \rightarrow D_{F}(x)\right]
$$

where $D_{F}(x)$ contains no nested phrase of the form

$$
\text { Vy }\left[\sim \in(y, A) \vee D_{B}(y)\right] \quad \text { or } \quad \exists y\left[\epsilon(y, A) \wedge D_{C}(y)\right] \text {. }
$$

$D_{F}(x)$ can be satisfied and still $x \in A$. Hence $P(A)$ will be strongly satisfied only by the entire data space. Also, if the collapsed form of the feature is $P(A) \equiv \exists x[\sim \in(x, A)$ $\left.\wedge_{D_{E}}(x)\right]$ its negation will be of form (9.1). Again, $D_{E}(x)$ can be satisfied and still $x \in A$. Hence $P(A)$ will be strongly satisfied only be sets consisting of the entire data space minus one point. Features which are strongly satisfied only by sets with a fixed number of points are of no interest here (except when "card(A)" is a primitive). Hence we insist that all formulae contain at least one phrase of form (9.2). ${ }^{1}$

Other elimination methods related to this discussion are described in Rothenberg (1973b).

1 The formulae in (9.2) intuitively specify conditions for membership of a point in $A$ (i.e., $\left.\forall y\left[\epsilon(y, A) \rightarrow D_{B}(y)\right]\right)$ or for non-membership of a point in $A$ (i. e., $\forall y\left[\sim D_{E}(y) \rightarrow \epsilon(y, A)\right]$. The existentially quantified formula in (9.2) may result from a negation of the universally quantified formula. 


\section{Models}

A formula is evaluated in the product set over which the vector of its free variables ranges. Thus if $S \subset U$ is temporarily our universal set, $P(A)$ is evaluated in $2^{S}$ (i.e., the power set of $\mathrm{S})^{1}$ and $\mathrm{D}(\mathrm{x}, \mathrm{y}, \mathrm{A}, \mathrm{B})$ is evaluated in $\mathrm{SXSX2}^{\mathrm{S}} \mathrm{X} 2 \mathrm{~S}$. (Note that formulae are never evaluated in the entire data space, $U$ (to be discussed).)

A model of the formula, $F_{\hat{i}}$, is an element of the product space (denoted by $\overline{\mathrm{S}}$ ), over which the vector of its free variables ranges, such that the substitution of each component of that element for the free variable satisfies the formula. The model is denoted by $M_{j}^{(\bar{S})}\left(F_{i}\right)$ where $\mathbf{j}$ indexes all such models (the ordering is arbitrary). When $\mathrm{F}_{\mathrm{i}}$ functions as a feature, it is denoted by $P_{i}$ and a model of it $M_{j}^{(\bar{S})}\left(P_{i}\right)$ (as distinct from a "realization", $M_{j}^{(\bar{S})}\left(F_{i}\right)$ ) must strongly satisfy $P_{i}$ in $\overline{\mathrm{S}}=2^{\mathrm{S}} \frac{\text { (see Section } 2) .}{(\overline{\mathrm{S}})} \mathrm{M}^{(\overline{\mathrm{S}})}\left(\mathrm{F}_{\mathrm{i}}\right)$ is the set of all models of a formula (called its extension) and $\overline{M^{(\tilde{S})}\left(P_{i}\right)}$ denotes the extension of feature, $P_{i}$.

The ease of satisfaction, $E\left(F_{i}\right)$, of a formula $F_{i}$, is defined as

$$
E\left(F_{i}\right)=\frac{\operatorname{card} \overline{\left(\overline{M(\bar{S})}\left(E_{i}\right)\right)}}{\operatorname{card}(\overline{\mathbf{S}})}
$$

("card" denotes the number of elements in the set within brackets).

The ease of satisfaction of a feature, $P_{i}$, is always defined in terms of satisfaction, not strong satisfaction (i.e., $\mathrm{E}\left(\mathrm{P}_{\mathbf{i}}\right)=\mathrm{E}\left(\mathrm{F}_{i}\right)$ ).

The overlap of a pair of formulae, $F_{i}$ and $F\left(\bar{S} j^{j}\right.$ is defined as $E\left(F_{i} \wedge F_{j}\right)$.

The following applies only to features: $\left|\mathrm{M}_{j}^{(\bar{S})}\left(P_{i}\right)\right|$ is the set of points of $s$ in the $j^{\text {th }}$ model of $P_{i}$ in $\vec{S}=2^{S}$. Let $n\left(P_{i}\right)=\operatorname{card}\left(M^{(\bar{S})\left(P_{i}\right)}\right)$. Then we define

$$
\left(M_{*}^{(\bar{S})}\left(P_{i}\right)\right)=\bigcup_{j=1}^{n\left(P_{i}\right)}\left|M_{j}^{(\bar{S})}\left(P_{i}\right)\right| .
$$

Restating our problem, we are given two disjoint classes of regions

$$
\begin{aligned}
& \overrightarrow{\mathrm{K}}=\left\{\mathrm{R}_{1}, \mathrm{R}_{2}, \ldots, \mathrm{R}_{\mathrm{N}}\right\} \\
& \overrightarrow{\mathrm{C}}=\left\{\mathrm{R}_{\mathrm{N}+1}, \mathrm{R}_{\mathrm{N}+2}, \ldots, \mathrm{R}_{\mathrm{N}+\mathrm{M}}\right\}
\end{aligned}
$$

and we wish to generate an ideal feature, $\bar{P}$, such that (see Section 1)

$$
\nabla k \exists_{j}\left(\left|M_{j}^{(\bar{U})}(\bar{P})\right|=R_{k}\right) \quad \text { where } R_{k} \in \bar{K}
$$

${ }_{A} \mathrm{~A}$ in $\mathrm{P}(\mathrm{A})$ is allowed to range only over subsets of $\mathrm{S}$. Points not in $\mathrm{A}$ and sets other than $A$, however, may lie anywhere in $U$. (In practice, in most cases, for purposes of computation reduction, points not in $S$ are restricted to the boundary of the complement of $S$ in $U$. 2 Note that $E\left(F_{i}\right)$ is generally defined with $\mathrm{S}=\mathrm{U}$. 
AND

$$
\forall \ell \forall j\left(\left|M_{j}^{(\bar{U})}(\bar{P})\right| \neq R_{\ell}\right) \quad \text { where } R_{\ell} \epsilon \overline{\mathrm{C}}
$$

Note that $\overline{\mathrm{K}} \subset \overline{\mathrm{M}^{(\overline{\mathrm{U}})}(\overline{\mathrm{P}})}$, When this inclusion is proper, $\overline{\mathrm{P}}$ is said to generalize $\overline{\mathrm{K}}$. We arbitrarily assign the subscripts of each given region, $R_{i}$. Now the subscripts of all $\mathrm{M}_{j}^{(\overline{\mathrm{U}})}\left(\mathrm{P}_{i}\right)$ are assigned such that

$$
\frac{1}{N} \sum_{j=1}^{N}\left(\operatorname{card}\left(R_{j} \cap\left|M_{j}^{(\bar{U})}\left(P_{i}\right)\right|\right) / \operatorname{card}\left(R_{j}\right)\right)
$$

is maximum for ali possible indexings (any such maximal indexing suffices).

\section{Measures of Success}

Let us denote formula $(10.1)$ as $\phi\left(\mathrm{P}_{i}, \overline{\mathrm{K}}\right)$ when all $\mathrm{R}_{j} \in \overline{\mathrm{K}}$ and as $\phi\left(\mathrm{P}_{i}, \overline{\mathrm{C}}\right)$ when all $R_{j} \in \bar{C}$. Let $R_{j}^{c}$ be the complement of $R_{j}$ in $U$ and let $\bar{K}^{c}$ be set of $R_{j}^{c}$ for all $j$ such that $\mathrm{R}_{\mathrm{j}} \in \overline{\mathrm{K}}$. Define $\gamma\left(\mathrm{P}_{\mathrm{i}}, \overline{\mathrm{K}}\right)=\phi\left(\mathrm{P}_{\mathrm{i}}, \overline{\mathrm{K}}\right)-\phi\left(\mathrm{P}_{\mathrm{i}}, \overline{\mathrm{K}} \mathrm{c}\right)$ and let

$$
\theta\left(\mathrm{P}_{\mathbf{i}}, \overline{\mathrm{K}}, \overline{\mathrm{C}}\right)=\gamma\left(\mathrm{P}_{\mathbf{i}}, \overline{\mathrm{K}}\right)-\gamma\left(\mathrm{P}_{\mathbf{i}}, \overline{\mathrm{C}}\right)
$$

be defined as the measure of success in segmentation. It will meas ure how well a feature approximates an ideal feature. Another such measure, called the success in partitioning is defined as

$$
\xi\left(\mathrm{P}_{i}, \overline{\mathrm{K}}_{,} \overline{\mathrm{C}}\right)=\frac{\operatorname{card}\left(\overline{\mathrm{K}} \cap \overline{\mathrm{M}^{(\overline{\mathrm{U}})}\left(\mathrm{P}_{i}\right)}\right)}{\operatorname{card}(\overline{\mathrm{K}})}-\frac{\operatorname{card}\left(\overline{\mathrm{C}} \cap_{\mathrm{M}}^{(\overline{\mathrm{U}})}\left(\mathrm{P}_{i}\right)\right)}{\operatorname{card}(\overline{\mathrm{C}})} .
$$

Other measures are also utilized (see Rothenberg, 1973b and Section 18).

Whenever feedback occurs, a set of weights, called reinforcements, on all features and derived predicates are accordingly altered. These are increased (or decreased) by adding, for example, $\xi\left(\mathrm{P}_{\mathrm{i}}, \overline{\mathrm{K}}, \overline{\mathrm{C}}\right)-.5+\left[\theta\left(\mathrm{P}_{\mathrm{i}}, \overline{\mathrm{K}}, \overline{\mathrm{C}}\right)-.5\right] / \mathrm{k}$ (where $\mathrm{k}$ is an integer) ${ }^{1}$ to the weights which are initially set at zero. This is done for all derived predicates ${ }^{2}$ and predicate forms which together form a successful (or unsuccessful) feature. Thus derived predicates and predicate forms accumulate reinforcement from a variety of features.

\section{Mutations}

We generate our features by starting with an arbitrarily generated feature, $P_{i}$, and successively altering its syntax by means of mutations which are functions which take one

$\overline{1}$ An optimal choice of $\mathrm{k}$ may be estimated by experimentation with the program. Actually any method of weighting $\xi\left(P_{i}, \bar{K}, \bar{C}\right)$ more heavily than $\theta\left(P_{i}, \bar{K}, \bar{C}\right)$ will suffice.

2 The negations of derived predicates are also treated as derived predicates and accumulate reinforcement accordingly. 
feature into another (i. e., $f_{n}\left(P_{i}\right)=P_{k}$ ). We specify three classes of mutations: restricting (iff $\overline{M^{(\bar{U})}\left(P_{k}\right)} \varsubsetneqq \overline{M^{(\bar{U})}\left(P_{i}\right)}$ ), relaxing (iff $\overline{M^{(\bar{U})}\left(P_{i}\right)} \varsubsetneqq \overline{M^{(\bar{U})}\left(P_{k}\right)}$ ), and neutral (otherwise).

Relaxing mutations (said to "relax" a formula) demonstrably include (Rothenberg, 1973b).

(1) Adding a subtree (i. e. derived or atomic predicate) below a node (on the graph of a distributive formula) which contains a universal quantifier.

(2) Eliminating a subtree below a node containing an existential quantifier.

(3) Replacing a universal quantifier by an existential quantifier (and restoring distributive form).

(4) Replacing " $\exists \mathrm{x} y$ " by " $\forall y \exists x$ " and restoring distributive form.

(5) Replacing a subformula (i.e. subtree) by one which it implies.

(6) Replacing $" \forall x(\epsilon[x, A] \vee D[x, \ldots]) "$ by $" \exists x(\epsilon[x, A] \wedge D[x, \ldots]) "$ when the former formula is not vacuous.

(7) Replacing $" \forall x(\epsilon[x, A] \vee D[x, \ldots]) "$ by $" \exists x(\sim \epsilon[x, A] \wedge D[x, \ldots])$ " when the former formula is not satisfied by the universal set.

The inverses of the above are restricting mutations (said to "restrict" a formula).

A neutral mutation consists of the replacement of a subtree on the graph of the distributive form of a feature by another subtree with the same free variables such that neither subtree repres ents a formula which implies the other. This includes permutations and exchanges of variables in a subformula. Other "composite" mutations are discussed in Rothenberg (1973b).

\section{Mutation Choice}

Here, among others, we will develop the following criteria: if given region $R_{j}$ is an element of $\bar{K}$, then (a) when $R_{j}$ does not satisfy $P_{i}$ a relaxing mutation on $P_{i}$ is required, and (b) when $R_{j}$ satisfies $P_{i}$ but not strongly a restricting mutation is required. When $R_{j}$ is an element of $\bar{C}$ and $R_{j}$ strongly satisfies $P_{i}$, either mutation may suffice (although a restricting mutation is usually preferred). These criteria are, of course, averaged for all elements of $\overline{\mathrm{K}}$ and $\overline{\mathrm{C}}$.

Assuming we have a feature, $\mathbf{P}_{\mathbf{i}}$, we now consider how to choose an appropriate mutation: Let $P_{i}$ be decomposed into a conjunction of two formulae, $P_{i}^{I}$ and $P_{i}^{D}$ such that $\mathrm{P}_{\mathrm{i}}^{\mathrm{I}}$ is data independent and $\mathrm{P}_{i}^{\mathrm{D}}$ contains data-dependent atomic predicates and only those data independent predicates which are inseparable from this portion of the formula (Rothenberg, $1973 b)$.

$$
P_{i}=P_{i}^{I} \wedge P_{i}^{D}
$$


The features are generated in form (13.1). Suppose we wish region $R_{j} \in \bar{K}$ to be a model of $P_{i}$ (note: strong satisfaction is required). Now let $S_{j}=2^{R_{j}} \cdot{ }^{\prime}$ Let "p $[\mathrm{A}]$ " represent the probability of event A. It has been proved (Rothenberg, 1973b) that if $R_{j}$ is a randomly selected region,

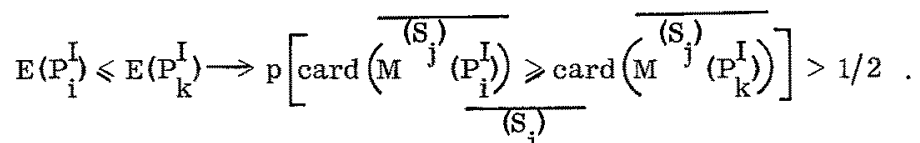

This holds only because all elements of $M{ }^{j}\left(P_{i}\right)$ are maximal realizations (i.e., models) of $P_{i}$ (13.2) can be intuitively seen if we consider the number of maximal: ${ }^{2}$ (1) circles, (2) convex regions and (3) "starlike" regions that may be contained in a region consisting of the interior of an arbitrary ink blot. Clearly the numbers decrease from (1) - (3) while the ease of satisfaction of the formulae (features) corres ponding to (1)-(3) increase. Let " $M_{L}{ }^{\left(S_{j}\right)}\left(P_{i}^{I}\right)$ " denote the largest (i.e. most points) model of $P_{i}^{I}$ in $S_{j}$. Then the following also holds:

$$
\begin{aligned}
& E\left(P_{i}^{I}\right)<E\left(P_{k}^{I}\right) \rightarrow p\left[\left|M_{L}^{\left(S_{j}\right)}\left(P_{i}^{I}\right)\right|=S_{j}\right]<p\left[\left|M_{L}^{\left(S_{j}\right)}\left(P_{k}^{I}\right)\right|=s_{j}\right] \\
& \left.\operatorname{card} \overline{\left(M^{\left(S_{j}\right)}\left(P_{i}^{I}\right)\right.}\right)>\operatorname{card} \overline{\left(M^{\left(S_{j}\right)}\left(P_{k}^{I}\right)\right)} \rightarrow p\left[E\left(P_{i}^{I}\right)<E\left(P_{k}^{I}\right)\right]>1 / 2
\end{aligned}
$$$$
\mathrm{E}\left(\mathrm{P}_{\mathbf{i}}^{\mathrm{I}}\right)<\mathrm{E}\left(\mathrm{P}_{\mathrm{k}}^{\mathrm{I}}\right) \rightarrow \mathrm{p}\left[\operatorname{card}\left(\left|\mathrm{M}_{\mathrm{L}}^{\left(\mathrm{S}_{j}\right)}\left(\mathrm{P}_{\mathrm{i}}^{\mathrm{I}}\right)\right|\right)<\operatorname{card}\left(\left|\mathrm{M}_{\mathrm{L}}^{\left(\mathrm{S}_{j}\right)}\left(\mathrm{P}_{\mathrm{k}}^{\mathrm{I}}\right)\right|\right)\right]>1 / 2
$$

$$
\operatorname{card}\left(\left|M_{L}^{\left(S_{j}\right)}\left(P_{i}^{I}\right)\right|\right)>\operatorname{card}\left(\left|M_{L}^{\left(S_{j}\right)}\left(P_{k}^{I}\right)\right|\right) \rightarrow p\left[E\left(P_{i}^{I}\right)>E\left(P_{k}^{I}\right)\right]>1 / 2
$$

The above indicates that if more than one object satisfies $P_{i}$ in $S_{j}$ or, if the largest model of $P_{i}$ in $S_{j}$ is smaller than desired, a relaxing mutation of $P_{i}$ is appropriate. Now we consider the case where $s_{j}=\left.2\right|_{j}\left|M_{j}^{(U)}\left(P_{i}^{D}\right)\right|$ for all $j$ (i.e., we evaluate $P_{i}^{I}$ in the space of all subsets of the $j^{\text {th }}$ model of $\mathrm{P}_{i}^{\mathrm{D}}$ ).

all $j$

$$
\text { Suppose for some } j,\left|M_{j}^{(U)}\left(P_{i}^{D}\right)\right| \cap R_{j} \neq R_{j} \text {. Then } P_{i}^{D} \text { will be relaxed until, for }
$$

$$
R_{j} \subset\left|M_{j}^{(U)}\left(P_{i}^{D}\right)\right|
$$

and such that

\footnotetext{
1 For brevity in notation, when models of features are denoted, the "-" over the super script will be omitted (e.g., $\mathrm{M}^{(\bar{U})}\left(\mathrm{P}_{i}\right)$ will be denoted by $\mathrm{M}^{(\mathrm{U})}\left(\mathrm{P}_{i}\right)$ ).

2 If we omit "maximal", clearly the ordering is the other way around.
} 


$$
\sum_{j=1}^{n\left(P_{i}\right)} \operatorname{card}\left(\left|M_{j}^{(U)}\left(P_{i}^{D}\right)\right|-R_{j}\right)
$$

is as small as possible. Note that it is possible that card $\left\{R_{\ell} \in \bar{K} \mid R_{\ell} \in S_{j}\right\}>1$ ("I| "means "such that"), $\mathbf{i}$, e., there are several $R_{\ell} \subset s_{j}$. Consider $p_{i}$ and $\bar{P}$ which is ideal feature. Let $M_{j}^{(U)}\left(P_{i}^{D}\right)=M_{j}^{(U)}\left(\bar{P}^{D}\right)=s_{j}$ (note: $R_{j} \subset\left|M_{j}^{(U)}\left(P_{i}^{D}\right)\right|$ ). Then it can be shown that (Rothenberg, 1973b):

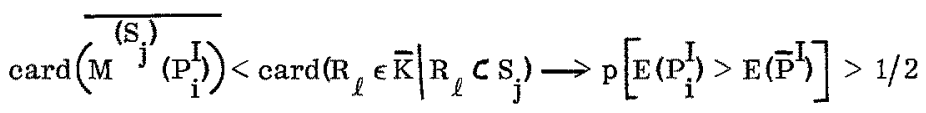

$$
\operatorname{card}\left(\left|M_{L}^{\left(S_{j}\right)}\left(P_{i}^{I}\right)\right|\right)>\operatorname{card}\left(R_{j}\right) \rightarrow P\left[E\left(P_{i}^{I}\right)>E\left(\bar{P}^{I}\right)\right]>1 / 2
$$

$$
E\left(P_{i}^{I}\right)>E\left(\bar{P}^{I}\right) \rightarrow p\left[\operatorname{card}\left(\mid M_{L}^{\left(S_{j}\right)}\left(P_{i}^{I}\right)\right)>\operatorname{card}\left(R_{j}\right)\right]>1 / 2
$$

That is, if there are two many models of $P_{i}^{I}$ relax $P_{i}^{I}$; if there are two many points to a given model of $\mathrm{P}_{i}^{\mathrm{I}}$, restrict $\mathrm{P}_{i}^{\mathrm{I}}$.

Suppose $\left|M_{j}^{(U)}\left(\bar{P}^{D}\right)\right| \neq\left|M_{j}^{(U)}\left(P_{i}^{D}\right)\right|$. Since we are here dealing with a size-invariant recognition system (intuitively, a system wherein the magnification of a geometric figures does not alter the features which it satisfies or fails to satisfy), the probable proportion of models in a space $s_{j}$ does not alter with the size of the space (after a certain minimal size). Hence (13.8) still holds (although (13.9 and (13.10) do not). Hence a comparison of how well (13.8) and (13.9) agree, i.e., the probability that

$$
\operatorname{card}\left(\overline{M^{\left(S_{j}\right)}\left(P_{i}^{I}\right)}\right)<\operatorname{card}\left(R_{\ell} \in \bar{K} \mid R_{\ell} \subset S_{j}\right) \leftrightarrow \operatorname{card}\left(\left|M_{L}^{\left(S_{j}\right)}\left(P_{i}^{I}\right)\right|\right)>\operatorname{card}\left(R_{j}\right)
$$

indicates how well $\left|\mathrm{M}_{j}^{(\mathrm{U})}\left(\overline{\mathrm{P}}^{\mathrm{D}}\right)\right|$ and $\left|\mathrm{M}_{j}^{(\mathrm{U})}\left(\mathrm{P}_{\mathrm{i}}^{\mathrm{D}}\right)\right|$ match. Because of (13.7), $\mathrm{E}\left(\overline{\mathrm{P}}^{\mathrm{D}}\right)$ can only be too large. Hence a failure of (13.11) indicates that $P_{i}^{D}$ should be restricted in accordance with (13.9), but without violating (13.7).

When $p_{i}^{D}$ is usually composed of one place atomic predicates (i.e., "gray scales"), mutations of $\mathrm{p}_{\dot{i}}^{\mathfrak{D}}$ are simpler than those on $\mathrm{P}_{\dot{i}}^{\mathrm{I}}$ and are performed first (when there is such a choice).

Other techniques similar to the above exist (Rothenberg, 1973b). Since probabalistic considerations are involved in the above, $(13.8)$ and (13.9) should be averaged over all $j$ (there are $\mathrm{N}$ such) and normalized; i.e., examine both.

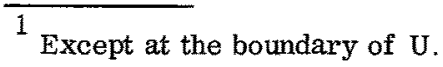




$$
\left.\mathrm{X}=\frac{1}{\mathrm{~N}} \sum_{\mathrm{j}=1}^{\mathrm{N}}\left(\left[\operatorname{card} \overline{\left(\mathrm{M}^{\left(\mathrm{S}_{\mathrm{j}}\right)}\left(\mathrm{P}_{\mathrm{i}}^{\mathrm{I}}\right)\right.}\right)-\operatorname{card}\left(\mathrm{R}_{\ell} \in \overline{\mathrm{K}} \mid \mathrm{R}_{\ell} \subset \mathrm{s}_{\mathrm{j}}\right)\right] / \operatorname{card}\left(\mathrm{R}_{\ell} \in \overline{\mathrm{K}} \mid \mathrm{R}_{\ell} \subset \mathrm{s}_{\mathrm{j}}\right)\right)
$$

and

$$
\mathrm{Y}=\frac{1}{\mathrm{~N}} \sum_{j=1}^{\mathrm{N}}\left(\left[\operatorname{card}\left(\left|\mathrm{M}_{\mathrm{L}}^{\left(\mathrm{S}_{\mathrm{j}}\right)}\left(\mathrm{P}_{\mathrm{i}}^{\mathrm{I}}\right)\right|\right)-\operatorname{card}\left(\mathrm{R}_{\mathrm{j}}\right)\right] / \operatorname{card}\left(\mathrm{R}_{j}\right)\right)
$$

and apply the strategies previously outlined according to whether $\mathrm{X}$ and $\mathrm{Y}$ are greater than zero.

We have assumed that each $R_{j}$ is randomly selected from subsets of $U$. This is not reasonable. However, since models of $P_{i}^{I}$ strongly s atisfy $P_{i}^{I}$, a region, $R_{j}$, which is not a model of $P_{i}^{I}$ need not be a model of $\sim \mathrm{p}_{i}^{i}$. Hence by applying our criteria for mutation choice to both formulae, $P_{i}^{I}$ and $\sim P_{i}^{I}$ (both of which for this purpose are treated as features), it appears that we may ameliorate our assumption of the randomness of $R_{j}$ (i.e., "models of $\mathrm{P}_{\dot{\mathrm{i}}}^{\mathrm{I}}$ are as random as models of $\left.\sim \mathrm{P}_{i}^{\mathrm{I}}\right)^{1}$. Thus the disagreement of criteria when applied to both $P_{i}^{I}$ and $\sim P_{i}^{I}$ (e.g., both $P_{i}$ and $\sim P_{i}$ require relaxing mutations) indicates the need for a neutral mutation. Notice that both measures of success (Section 11) are reflected in the above. (Details are discussed in Rothenberg (1973b).)

\section{Nodified Quantifiers}

Now a more powerful technique of mutation selection is described; note that all quantified point variables in a formula $P(A)$ range over either the set $A$ (e.g., are restricted by " $\in(\mathrm{x}, A)$ " within the scope of the quantifier) or the complement $A^{c}$ of $A$, or range over the universal set (when no set membership atomic predicate restricts its range). Thus when, on the graph of a formula, a node containing a quantifier has no branch containing a set membership atomic predicate, the quantified variable ranges over the universal set (or its power set if the variable is a set variable). (Note that in subformula $\forall x(\sim \epsilon[x, A] \vee F[x, \ldots]) \quad x$ ranges over $A$ and in subformula $\forall x(\epsilon[x, A] \vee F[x, \ldots])$, $x$ ranges over $A$. However, when the subformula is $\exists x(\epsilon[x, A] \wedge F[x, \ldots])$, $x$ ranges over A, etc. $)^{2}$.

Let $r_{q}$ denote the set over which variable $x_{q}$ ranges (where $q$ indexes the variables in a nested form - see Section 3). ${ }^{3}$ Since we are dealing with a size invariant system (see

${ }_{1}$ That is, if this randomness with respect to the above criteria does not hold, it appears that in these cases, we will know it because of opposite indications.

2 Note that

$$
\forall x(\sim \in[x, A] \vee F[x, \ldots]) \equiv \underset{x \in A}{\forall x}(F[x, .]) \text { and } \exists x(\in[x, A] \vee F[x, \ldots]) \equiv \underset{x \in A}{\exists x}(F[x, \ldots])
$$

etc.

${ }^{3} \Gamma_{q}$ always is one of $A, A^{c}, U, 2^{A}$, or $2^{A^{C}}$. 
previous section) we here consider the fraction of $x_{q}$ in $\Gamma_{q}$ (rather than the fraction of $x_{q}$ in U) for which a formula is satisfied. (Similar remarks apply to set variables.) ${ }^{1}$

The following notation will be utilized: all distributive forms in the distributive set will be arbitrarily indexed and $\mathrm{P}_{\mathbf{i}}^{(\mathrm{k})}$ will indicate the $\mathrm{kth}$ such distributive form of feature $P_{i}$. It can be shown that an arbitrary indexing of quantifiers in a prenex form from which the distributive set derives (see Section 4) will result in a corresponding ordering of the quantifiers in each distributive form. This follows from the following, which are consequences of the definition of the distributive set and of distributive form (see Section 4 ).

(a) If, in placing a prenex formula into a distributive form, one quantifier in the prenex formula becomes more than one quantifier (using the same variable) in the distributive form (e.g., $\forall x\left(F_{1}(x) \wedge F_{2}(x)\right)$ becomes $\left.\forall x\left(F_{1}(x)\right) \wedge \forall x\left(F_{2}(x)\right)\right)$, this occurs in all distributive forms in the distributive set.

(b) If two or more quantifiers using distinct variables in a prenex formula become quantifiers using the same variable in a distributive form (e. $\mathrm{g}$. , $\forall x \exists y\left(F_{1}(x) \wedge F_{2}(y)\right)$ becomes $\left.\forall x\left(F_{1}(x)\right) \wedge \exists x\left(F_{2}(x)\right)\right)$, the number of quantifiers in the prenex form and in the distributive form are equal.

Note that in (a) above the number of quantifiers increases and the number of variables remains unchanged. In (b) the number of variables is reduced, but the number of quantifiers remains unchanged.

Hence all distributive formulae in a distributive set have the same number of

quantifiers. All nested formulae (see Section 4) have the same number of quantifiers as the prenex formula (but possibly fewer variables). However, each of the distributive formulae may have more quantifiers than the prenex formula (even when the distributive formulae have fewer variables). Hence two or more quantifiers using the same variable in a distributive formula may have the same index las derived from the indexing of quantifiers in the prenex formula). Such quantifiers will be abritrarily ordered. That same ordering will be maintained in all distributive formulae, each of whose quantifiers will be subscripted by this ordering.

Let $Q_{r}^{(k)}$ denote the $r^{\text {th }}$ quantifier in $\mathrm{P}_{i}^{(k)}$ (i.e., the $\mathrm{k}^{\text {th }}$ distributive form of $\mathrm{P}_{i}$ ), $x_{r}^{(k)}$ denote the variable quantified by $Q_{r}^{(k)}$ and $F_{r}{ }_{r}$ denote the subformula within its scope (i. e., its subtrees on the graph of $\left.\mathbf{P}_{i}^{(\mathrm{k})}\right)^{\mathbf{r}}$. Simple methods exist (Rothenberg, 1973b) which guarantee that the subscripting of two or more quantifiers of the same variable is consistent across all distributive forms in the sense that, if they have the same subformula within

\footnotetext{
${ }^{1}$ When $\Gamma_{q}=U-S_{j}$; for computational economy, $x_{q}$ is allowed to range only over points in the boundary of the complement of $\mathrm{S}$. Note that no formula is ever evaluated in $U$.
} 
their scope, they bave the same subscript. Note that each variable, $x_{r}^{\langle(k)}$, is subscripted according to its quantifier's subscript, and hence the same variable may have different subscripts when separately quantified (i.e., such quantifiers are on the same level of the graph of $\mathrm{P}_{\mathrm{i}}^{(\mathrm{k})}$ ). If $\mathrm{x}_{\mathrm{p}}^{(\mathrm{k})}$ and $\mathrm{x}_{\mathrm{r}}^{(\mathrm{k})}$ are two such variables we will denote their equivalence by $" x_{p}^{(k)}=x_{r}^{(k)} "$. (Note that such variables are not separately quantified in nested formulae.) $\Gamma_{p}^{(k)}$ will denote the set over which $x_{p}^{(k)}$ ranges (if $x_{p}^{(k)}$ is not a point variable, $\Gamma_{p}^{(k)}$ denotes the power set over which $x_{p}^{(k)}$ ranges).

Let " $\mathrm{Q}_{\mathrm{p}}^{(\mathrm{k})}{ }_{S Q_{\mathrm{r}}}^{(\mathrm{k})}$ " denote that $\mathrm{Q}_{\mathrm{p}}^{(\mathrm{k})}$ is a successor node of $\mathrm{Q}_{\mathrm{r}}^{(\mathrm{k})}$ in $\mathrm{P}_{\mathrm{i}}^{(\mathrm{k})}$ (i.e., $\mathrm{Q}_{\mathrm{r}}^{(\mathrm{k})}$ is within the scope of $\left.Q_{p}^{(k)}\right)$. "Q $Q_{p}=\nabla^{\prime \prime}$ and " $Q_{p}=3^{\prime \prime}$ will indicate, respectively, that $Q_{p}$ is a universal quantifier and that $Q_{p}$ is an extential quantifier (note that the superscript is unnecessary because of the correspondence in indexing across distributive forms of a formulal. Superscripts will henceforth be included only when relevant or useful for purposes of clarity. Given a region, $R_{q}$, and a feature, $P_{i}$, note that " $R_{q}=\left|M{ }^{\prime}{ }^{\left(P_{i}\right)}\right| "$ (subscript is omitted because irrelevant) denotes strong satisfaction of $P_{i}$ by $R_{q}$ in space, $S_{j}$, and
(S) $" \mathrm{R}_{\mathrm{q}}=\left|\mathrm{M}^{\left(\mathrm{S}_{\mathrm{j}}\right)}\left(\mathrm{F}_{\mathrm{i}}\right)\right| "$ denotes satisfaction but not necessarily strong satisfaction. ${ }^{1}$

Now consider a feature, $P_{i}(A)$, and two given regions $R_{q} \in \bar{K}$ and $R_{t} \in \bar{C}$. Suppose we wish to alter $P_{i}$ so that $R_{q}$ strongly satisfies the altered feature and $R_{t}$ does not. ${ }^{2}$ From the preceding section we see that, in general:
(a) if $R_{q} \neq\left|M^{\left(S_{j}\right)}\left(F_{i}\right)\right|$
(i. e., $R_{q}$ fails to satisfy $P_{i}$ )

a relaxing mutation is required,
(b) If $R_{q}=\left|M^{\left(S_{j}\right)}\left(F_{i}\right)\right|$ but $R_{q} \neq\left|M^{\left(S_{j}\right)}\left(P_{i}\right)\right|$
(i. e., $R_{q}$ satisfies $P_{i}$ but not strongly),

a restricting mutation is required,
(c) if $R_{t}=\left|M^{\left(S_{j}\right)}\left(P_{i}\right)\right|$

either a neutral mutation or a mutation according to (a) or (b) above is required. (Note: $\mathrm{R}_{\mathrm{t}} \in \overline{\mathrm{C}}-$ to be discussed in Section 15.)

Combinations of (a), (b) and (c) will also be discussed in the next section.

\footnotetext{
${ }^{1}$ Note that it is here assumed that $R_{q} \subset S_{j}$. ${ }^{2}$ As in the previous section, $\mathrm{P}_{\mathbf{i}}^{\mathrm{I}}$ and $\mathrm{P}_{\mathbf{i}}^{\mathrm{D}}$ are independently mutated (although this is not
explicitly noted here).
} 
First we consider case (a) above Let $A=R_{q}$ ( $A$ is the free set variable in $P_{i}(A)$ ). Note that $A$ is no longer a free variable Arbitrarily select a distributive form, $P_{i}^{(k)}$. Now select $p$ such that $Q_{p}=\forall$ and such that for all quantifiers $Q_{r}^{(\ell)}$ (in all distributive forms, $\mathrm{p}_{i}^{(\ell)}$

$$
\sum_{\ell}\left(\operatorname{card}\left(Q_{r}^{(\ell)} \mid\left(Q_{r}^{(\ell)} s Q_{p}^{(\ell)}\right) \wedge\left(Q_{r}^{(\ell)}=\nabla\right)\right)\right.
$$

is maximum (i.e., such that $Q_{p}$ has the maximum number of successor nodes which are universal quantifiers, summed over all distributive forms in the distributive set of $P_{i}$ ). If two or more quantifiers are equivalent in this respect, an arbitrary selection will suffice.

Now replace $Q_{p}$ by the modified quantifier, $\bar{Q}_{\mathrm{p}}$, which computes $\bar{K}_{\mathrm{p}}$, which is defined as that fraction of the $x_{p}$ 's in $\Gamma_{p}$ for which $P_{i}$ is true (i.e., for which $R_{q}=$ $M^{\left(S_{j}\right)}\left(F_{i}\right) \mid$; which definition is clarified by the following procedure: every subformula of $P_{i}$ is of the form $Q_{p} x_{p}\left(F_{p}\left[x_{1}, \ldots, x_{p}\right]\right)$ (although it may be necessary to re-index the quantifiers in the distributive form so that only variables in $F_{p}$ are indexed-see Section 4). Let each element of $\Gamma_{n}$ be denoted by $c_{n}\left(k_{n}\right)$ where $k_{n}$ indexes all elements of $\Gamma_{n}$. Define $K\left(c_{1}\left(k_{1}\right), c_{2}\left(k_{2}\right), \ldots, c_{p-1}\left(k_{p-1}\right)\right)$ as the fraction of substitutions of the elements of $r_{p}$ for $x_{p}$ for which $P_{i}(A)$ is true when $\left(x_{p}, \ldots, x_{p-1}\right)=\left(c_{1}\left(k_{1}\right), \ldots, c_{p-1}\left(k_{p-1}\right)\right)$. For all $n \leq p-1$, if $Q_{n}=V$, define $K\left(c_{1}\left(k_{1}\right), \ldots, c_{n-1}\left(k_{n-1}\right)\right)=\min _{k_{n}}\left(K\left(c_{1}\left(k_{1}\right), \ldots, c_{n}\left(k_{n}\right)\right) ;\right.$ if $Q_{n}=\exists$, then $K\left(c_{1}\left(k_{1}\right), \ldots, c_{n-1}\left(k_{n-1}\right)\right)=\max _{k_{n}}\left(K\left(c_{1}\left(k_{1}\right), \ldots, c_{n}\left(k_{n}\right)\right)\right.$. When $n=1$, by definition, $\bar{K}_{p}=k$. However, if when $\bar{K}_{p}=0, R_{q}$ still fails to satisfy $P_{i}$, by definition $\bar{K}_{p}=-\infty .^{1}$ Note that $\bar{K}_{p}$ is the same regardless of the distributive form chosen (i.e., regardless of the $k$ in $F_{p}^{(k)}$ ). Actually, the procedure will operate on a prenex form (it is best to use nested form).

Now we replace $Q_{p}$ by another modified quantifier, $\hat{Q}_{p}^{(k)}$, which simi larly computes $\hat{K}_{p}^{(k)}$, which is defined as that fraction of the $x_{p}^{(k)} / s$ in $\Gamma_{p}^{(k)}$ for which $F^{(k)}$ is satisfied, averaged over all substitutions of constants for the free variables in $F_{p}^{(k)^{p}}$. Note that the distributive form chosen is now relevant and the superscript of $\hat{\mathrm{K}}_{\mathrm{p}}^{\mathrm{k}}(\mathrm{p})$ is necessary. (Superscripts of $x_{p}^{(k)}, \Gamma_{p}^{(k)}$ and $Q_{p}^{(k)}$ are included for clarity.) That is, $\bar{K}_{p}^{(k)}$ is such that feature $P_{i}$ is satisfied and $\hat{K}_{p}^{(k)}$ is such that subformula $F_{p}^{(k)}$ is satisfied. Hence always $\hat{\mathrm{K}}_{\mathrm{p}}^{(\mathrm{k})} \geq 0$.

Note that $\overline{\mathrm{Q}}_{\mathrm{p}}$ and $\hat{Q}_{\mathrm{p}}^{(\mathrm{k})}$ ' are correctly interpreted as "for $\overline{\mathrm{K}}_{\mathrm{p}} \cdot 100$ percent of $\mathrm{x}_{\mathrm{p}}$ " and as

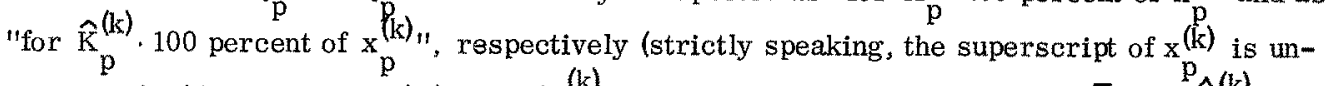
necessary). Also note that $E\left(P_{i}\right)$ and $E\left(F_{p}^{(k)}\right)$ are increased by replacing $Q_{p}$ by $\bar{Q}_{p}$ and $\hat{Q}_{p}^{(k)}$, respectively. $\bar{K}_{p}$ and $\widehat{K}_{p}^{(k)}$ are called the values of their respective quantifiers $\left(\bar{Q}_{p}^{p}\right.$ and $\left.\hat{Q}_{p}^{(k)}\right)$.

\footnotetext{
${ }^{1}$ This can be built into the above algorithm.
} 
Now define $\overline{\mathrm{D}}_{\mathrm{p}}=\overline{\mathrm{K}}_{\mathrm{p}}-1$. Restore the original quantifier, $\mathrm{Q}_{\mathrm{p}}$, and select another universal quantifier, $Q_{r}$, according to condition (14.1). Again, replace $Q_{r}$ by $\bar{Q}_{r}$ and $\hat{Q}_{r}(k)$ and compute the corresponding values of $\bar{K}_{r}, \hat{K}_{r}^{(k)}$ and $\bar{D}_{r}$. Restore $Q_{r}$ and repeat the procedure till all universal quantifiers in $P_{i}^{(k)^{r}}$ are exhausted. (This can be done in a single computation (Rothenberg, 1973b).)

Suppose for some $p, \bar{K}_{p}=-\infty$. Using $\bar{Q}_{p}$ is defined as equivalent to replacing $F_{p}^{(k)}$ by a tautology. A value of "1" in the numerator of $\bar{K}_{p}$ (i.e., $\bar{K}=1 / \operatorname{card}\left(\Gamma_{p}\right)$ ) is equivalent to replacing $Q_{p}$ by an existential quantifier. When $\bar{K}_{p}^{p}=-\infty$ we may conclude that no relaxing mutation within $F_{p}^{(k)}$ will result in $R_{q}=\left|M^{\left(S_{j}\right)}\left(F_{i}\right)\right|$ (or, of course, in $R_{q}=\left|M^{\left(S_{j}\right)}\left(P_{i}\right)\right|$ ). (Hence modifications of quantifiers which are successors of $Q_{p}$ on any tree of any distributive form in the distributive set may be avoided.) Note, however, that replacing a universal by an existential quantifier may increase $E\left(F_{p}^{(k)}\right)$ (and hence $E\left(F_{i}\right)$ ) less than a mutation which adds a subformula within $\mathrm{F}_{\mathrm{p}}^{(\mathrm{k})}$. Therefore when $\overline{\mathrm{K}}_{\mathrm{p}}=0$ (i.e. $-\infty<\overline{\mathrm{K}}_{\mathrm{p}}<1 /$ card $\left(\Gamma_{\mathrm{p}}\right.$ )) we may not without some hazard avoid considering the modification of $Q_{r} \mid \exists\left(Q_{r}^{(\ell)} Q_{p}^{(\ell)}\right)_{p}^{p}$.

Now consider case (b) where $R_{q}$ satisfies $P_{i}$ but not strongly. The procedure is similar: Again, arbitrarily select a distributive form, $P_{i}^{(k)}$ and let $A=R_{q}$. Select $p$ such that $Q_{p}=\exists$ and such that (similarly to $(14.1)$ )

$$
\sum_{\ell}\left(\operatorname{card} Q_{r}^{(\ell)} \mid\left(Q_{r}^{(\ell)} s Q_{p}^{(\ell)}\right) \wedge\left(Q_{r}^{(\ell)}=\exists\right)\right)
$$

is maximum.

Replace $Q_{p}$ by $\bar{Q}_{p}$, and compute $\bar{K}_{p}$ which is defined as previously except that if, for all $k$, no replacement of $F_{p}^{(k)}$ by a contradiction (i.e., a formula which is always false) reqults in $R_{q} \neq M\left(F_{i}\right)^{1}$ by definition, $\bar{K}_{p}=\infty$. We then replace $Q_{p}$ by $\overline{\bar{Q}}_{p}$ which computes $\overline{\bar{K}}_{p}$ which is defined similarly to $\bar{K}_{p}$ except that we now insist that $R_{q}=M\left(P_{i}\right)$ (strong satisfaction) instead of $R_{q}=M\left(F_{i}\right)$ (satisfaction but not strong satisfaction). Note that $\bar{K}_{p}$ and $\overline{\bar{K}}_{p}$ are not necessarily equal when $K_{p}=\infty$.

Now we again replace $Q_{p}$ by the modified quantifier $\hat{Q}_{p}^{(k)}$ which computes $\hat{K}_{p}(k)$ which is defined (as previously) as the fraction of the $x_{p}^{(k)} / s$ in $\Gamma_{p}^{(k)}$ for which $F_{p}^{(k)}$ is satisfied, averaged as before $\left(1 / \operatorname{card}\left(\Gamma_{p}^{(k)} \leq \hat{K}_{p}^{(k)} \leq 1\right)\right)$. Note that $E\left(P_{i}\right)$ and $E\left(F_{p}\right)$ are decreased by replacing $Q_{p}$ by the modified quantifiers (here, always, $\bar{K}_{p} \geq 1 / \operatorname{card}\left(\Gamma_{p}\right)$ ).

Now define $\overline{\mathrm{D}}_{\mathrm{p}}=\overline{\mathrm{K}}_{\mathrm{p}}$. Restore the original quantifier, $\mathrm{Q}_{\mathrm{p}}$, select another existential quantifier according to (14.2) and repeat the above procedure until all existential quantifiers in $\mathrm{P}_{i}^{(\mathrm{k})}$ are exhausted. (Again this can be done in a single computation (Rothenberg, 1973b).)

The superscript of "M ${ }^{\left(S_{j}\right)}\left(F_{i}\right)$ " is henceforth omitted in such equations when $R_{q} \subset S_{j}$. 
Note that when $\overline{\mathrm{K}}_{\mathrm{p}}=\infty$ or $\overline{\overline{\mathrm{K}}}_{\mathrm{p}}=\infty$, we may conclude that no restricting mutation within $F_{p}^{(k)}$ for any $k$ (i.e., any distributive form) will result in $R_{q}=M\left(F_{i}\right)$ or $R_{q}=M_{i}\left(P_{i}\right)$ respectively. When $\bar{K}_{p}=1$ (or $\overline{\bar{K}}_{p}=1$ or $\hat{K}_{p}^{(k)}=1$ ) we are, in effect, replacing an existential by a universal quantifier. Note, however, that replacing an existential by a universal quantifier may decrease $\left.\mathrm{E}_{\mathrm{p}}^{(\mathrm{k})}{ }_{\mathrm{p}}\right)$ less than a mutation which adds a subformula within $\mathrm{F}_{\mathrm{p}}^{(\mathrm{k})}$. Hence, in this case, we may not without some hazard avoid considering the modification of $Q_{r} \mid \exists \ell\left(Q_{r}^{(\ell)} Q_{p}^{(\ell)}\right)$.

With appropriate modifications the above procedure will operate on quantifiers of set (as well as point) variables,

We now define the degree of satisfaction, $D\left(P_{i}, R_{q}\right)$, of $R_{q}$ with respect to feature, $P_{i^{3}}$ as

$$
D\left(P_{i}, R_{q}\right)=\max _{p}\left(\bar{D}_{p}\right) \mid A=R_{q}
$$

("A" is the free variable in " $P_{i}(A) "$ ")

When $D\left(P_{i}, R_{q}\right)$ is negative (universal quantifiers have been modified because $\left.\mathbf{R}_{\mathrm{q}} \neq \mathrm{M}\left(\mathrm{F}_{\mathbf{i}}\right)\right), \quad \mathrm{D}\left(\mathrm{P}_{\mathbf{i}}, \mathrm{R}_{\mathrm{q}}\right)$ is called the degree of undersatisfaction of $\mathbf{R}_{\mathrm{q}}$ with respect to $\mathbf{P}_{\mathrm{i}}$. When $D\left(P_{i}, R_{q}\right)$ is positive (existential quantifiers have been modified because $R_{q}=M\left(F_{i}\right)$ ) but $R_{q} \neq M\left(P_{i}\right), \quad D\left(P_{i}, R_{q}\right)$ is called the degree of oversatisfaction of $R_{q}$ with respect to $\mathbf{P}_{\mathbf{i}}$

In our probelm here we wish to generate a feature, $P_{i}$, such that $R_{k} \in \bar{K} \rightarrow$ $R_{k}=M\left(P_{i}\right)$ and $R_{\ell} \in \bar{C} \longrightarrow R_{\ell} \neq M\left(P_{i}\right)$. Accordingly we define the satisfaction gap, $G\left(P_{i}\right)$, of $\mathrm{P}_{i}$ as

$$
G\left(P_{i}\right)=\min _{R_{k} \in \bar{K}}\left(D\left(P_{i}, R_{k}\right)\right)-\max _{R_{\ell} \in \bar{C}}\left(D\left(P_{i^{\prime}}, R_{\ell}\right)\right)
$$

Note the correspondence between $G\left(P_{i}\right)$ and our "measures of success" (Section 11 ). Although we are seeking strong satisfaction while $G\left(P_{i}\right)$ deals with satisfaction which is not necessarily strong (appropriate adjustements can be made in the definition of $G\left(P_{i}\right)$ ), it will be seen that $G\left(P_{i}\right)$ has uses other than measuring the success of $P_{i}-$ see Section 17 . Note also that modified quantifiers of the $F_{p}^{(k)}$ in $P_{i}$ correspond to mutations of such subformulas both in kinds and amounts of alterations in ease of satisfaction, $\mathrm{E}\left(\mathrm{P}_{i}\right)$ and $\mathrm{E}\left(\mathrm{F}_{\mathrm{p}}^{(\mathrm{k})}\right)$. Also, the alterations in formulae due to quantifier modification are related by implication just as are the alterations due to mutations.

\footnotetext{
1 Note the intuitive interpretation of these notions-e.g., of two concave (i.e., non-convex) regions (e.g., (2) and $\mathcal{O}$ ), one may be "more concave" (the former) than the other. Experimentation will reveal that the above techniques preserve such intuition (e.g., 2 ) and (0) are approximately "equally concave")-see Rothenberg (1973b).
} 
15. Ease of Satisfaction; Mutation

It is easily seen that, when $\hat{K}_{p}^{(k)}$ is evaluated for all $R_{i} \in \bar{K}$ and all $R_{j} \in \overline{\mathrm{C}}$ and $\hat{\mathrm{K}}_{p}^{*}(\mathrm{k})$ is defined as an average of all such $\hat{\mathrm{K}}_{\mathrm{p}}(\mathrm{k}$,

$$
\mathrm{E}\left(\mathrm{F}_{\mathrm{p}}^{(\mathrm{k})}\right) \approx \hat{\mathrm{K}}_{\mathrm{p}}^{*}(\mathrm{k}) . \quad(" \approx " \text { means "approximately equal to") }
$$

$" \approx "$ is used instead of $"="$ in the above beeause the probability of such equivalence depends upon and sharply increases as the number of regions $\left(R_{i}\right.$ and $\left.R_{j}\right)$ for which $\hat{K}_{p}^{(k)}$ is evaluated and averaged increases.

All modified quantifiers are estimated in a single evaluation of $P_{i}$ by considering all substitutions for the variables in the formula (regardless of whether the variables are quantified by universai or existential quantifiers). Such methods (which result in estimates for $E\left(F_{p}^{(k)}\right)$ rather than exact values) are essential to reduce computation to feasible proportions. Hence, when $P_{1}$ is evaluated, we obtain an estimate of the ease of satisfaction $E\left(F_{p}^{(k)}\right)$ of all its subformulae, $F_{p}^{(k)}$. Some such subtrees (on the graph of $P_{i}$ ) become derived predicates after the simple abstraction procedure is applied. When these derived predicates are stored, their available estimated eases of satisfaction are also retained.

Each of the $\overline{\mathrm{K}}_{\mathrm{p}}$ (and $\overline{\overline{\mathrm{K}}}_{\mathrm{p}}$ ) are also averaged for all $\mathrm{R}_{i} \in \overline{\mathrm{K}}$ (in cases (a) and (b)) or for all $R_{j} \in \bar{C}$ (in case (c) - to be discussed). Let $\vec{K}_{p}$ (and $\overrightarrow{\mathrm{K}}_{\mathrm{p}}$ ) represent such averages. Then a mutation of subformula $F_{p}^{(k)}$ (of $P_{\hat{i}}$ ) which results in another subformula, $\mathrm{F}_{\mathrm{p}}(\mathrm{k})$, such that $\mathrm{E}\left(\mathrm{F}_{\mathrm{p}}^{(\mathrm{k})}\right)=\overline{\mathrm{K}}_{\mathrm{p}}$ (or $\overline{\mathrm{K}}_{\mathrm{p}}$, if appropriate) may result in transforming $\mathrm{P}_{i}$ into an ideal feature. No other mutation of $F_{p}^{(k)}$ can achieve this result. Hence mutations are chosen so that new subformulae $\left(\breve{F}_{\mathrm{p}}^{(\mathrm{k})}\right)$ result where, as closely as possible, $\mathrm{E}\left(\breve{\mathrm{F}}_{\mathrm{p}}^{\mathrm{p}}(\mathrm{k})=\stackrel{\mathrm{K}}{\mathrm{K}}_{\mathrm{p}}\right.$. For this purpose, the $F_{p}^{(k)}$ in $P_{i}$ are compared with the derived predicates previously extracted and, in general, in much simplified form, the procedure is:

(1) Determine whether a relaxing, restricting or neutral mutation (to be discussed) is required.

(2) Examine that distributive form, $\mathbf{p}_{i}^{(k)}$, of the feature, $\mathbf{P}_{i}$, to be mutated (usually that feature with the highest measure of success) which, when compared (by the simple abstration procedure) to other features previously generated, yields a derived predicate whose formula is the longest of those of derived predicates so obtained ( $k$ is thus chosen to facilitate the comparisons (in the abstraction procedures) of the new feature generated with previously generated features). When we evaluate $\mathrm{P}_{i}(\mathrm{~A})$ we thereby compute $\overrightarrow{\mathrm{K}}_{\mathrm{p}}$ and $\widehat{\mathrm{K}}_{\mathrm{p}}(\mathrm{k})$ for all p. At first the collapsed form (see Section 5) of $\mathrm{P}_{1}^{(\mathrm{k})}$ is examined.

I More refined methods than averaging are used; see next section. 
(3) Compare each subformula, $\underset{\mathrm{F}}{\mathrm{v}}(\mathrm{k})$, which would result from each mutation of the required type (on a corresponding subformula $F_{p}^{(k)}$ of $P_{i}$ ) with each of the derived predicates which has been stored. Comparison prior to mutation avoids the examination of many possibilities (see Rothenberg, 1973b). Also, of mutations which add subformulae within the scope of a quantifier (see Section 12) only the addition of such subformulae as are included in the list of derived and atomic predicates are considered.

(4) Select that mutation which produces a subformula, $\underset{\mathrm{F}}{\mathbf{v}(k)}$ (of the new feature), which is identical to a derived predicate whose ease of satisfaction most closely approximates that required (i. e., $\mathrm{E}\left(\mathrm{F}_{\mathrm{p}}^{\mathrm{k}}\right) \approx \mathrm{K}_{\mathrm{p}}$; see above discussion). ${ }^{1}$ If there are several such mutations, select that which results in the most reinforced (i.e. with the highest (reinforcement" - see Section 11) derived predicate. If none such exists, decompress (see Section 5) $\mathrm{P}_{i}^{(\mathrm{k})}$ one layer and try again. If repeated decompression fails, try another distributive form of $\mathrm{P}_{\mathbf{i}}$. If this also fails:

(5) Compare the features to be altered, $\mathrm{P}_{\mathbf{i}}$, with each of the predicate forms (see Section 8) which have been stored. Select a predicate form (if such exists) which resembles $P_{i}$ (or one of its subformulae) in the sense that, if corresponding subformulae of $P_{i}$ (which must have the same free variables as the dummy predicate) replace the dummy predicates, a formula results which constitutes a mutation of $P_{i}$ of the required type. If such resulting formula contains another dummy predicate, replace it by the most reinforced derived predicate (or atomic predicate) with the same free variables - see last part of Example 6 below. If a choice of predicate forms exists, select that with the highest reinforcement. If no such predicate form exists, a mutation of the required type is chosen at random and the footnote below applies.

Criteria restricting such random choice exist. The details (Rothenberg, 1973b) of the above procedures are constructed so that it is extremely unlikely that random choice will be necessary (except extremely rarely) after the initial stages of the learning procedure (note that, almost always, some mutation will better approximate the required ease of satisfaction of a subformula of $\mathrm{P}_{1}$ than another). It is, of course, possible (in some applications) to construct (from certain atomic predicates) inherently two-valued predicates (such as that a set must contain an even number of points) so that quantifier modification cannot guide the mutation procedure. In this case, techniques which develop those of Section 13 must be used and much greater use of random choice results.

\footnotetext{
1 If the ease of satisfaction of a derived predicate (or subformula resulting from a possible mutation) is not available by the methods described, it may be estimated by valuations of the formula in randomly chosen small spaces (as well as by other estimating techniquessee Rothenberg, 1973b).
} 


\section{$\underline{\text { Example } 6}$}

Suppose we wish to relax $P_{21}(A)$, which asserts that the region substituted for $A$ has a point which is "between" "no two other points in the region (i.e., a "cusp" if the region is connected). Let $\mathrm{D}_{14}(\mathrm{x}, \mathrm{y}) \equiv \forall \mathrm{z}\left[\sim \in(\mathrm{z}, \mathrm{A}) \vee \sim \mathrm{D}_{\mathrm{b}}(\mathrm{y}, \mathrm{x}, \mathrm{z})\right]$ and let $\mathrm{D}_{\mathrm{b}}(\mathrm{y}, \mathrm{x}, \mathrm{z})$ (previously defined) be in our list of derived predicates so that the collapsed form of our chosen distributive form of $\mathrm{P}_{21}(\mathrm{~A})$ is

$$
\mathrm{P}_{21}(\mathrm{~A})=\underset{\uparrow}{\exists \mathrm{x}}\left[\epsilon(\mathrm{x}, \mathrm{A}) \vee \uparrow_{1} \stackrel{\mathrm{Q}}{\mathrm{Q}}_{2}\left(\sim \in[\mathrm{y}, \mathrm{A}] \vee \mathrm{D}_{14}[\mathrm{x}, \mathrm{y}]\right)\right]
$$

Suppose we wish a relaxing mutation and our list of derived predicates contains $D_{a}(x, y)$ (previously defined) and

$$
D_{15}(x) \equiv \forall y\left[\sim \in(y, A) \vee D_{a}(x, y) \vee D_{14}(x, y)\right]
$$

and $\mathrm{E}\left(\mathrm{D}_{15}(\mathrm{x})\right) \approx \frac{\mathrm{*}}{\mathrm{K}_{2}}$ (which corresponds to $\mathrm{Q}_{2}$ in the distributive form above). Then our mutation (of type 1 - Section 12) yields a new feature,

$$
\breve{\mathrm{P}}(\mathrm{A}) \equiv \exists \mathrm{x}\left[\epsilon(\mathrm{x}, \mathrm{A}) \vee \forall y\left(\sim \in[\mathrm{y}, \mathrm{A}] \vee \mathrm{D}_{\mathrm{a}}[\mathrm{x}, \mathrm{y}] \vee \mathrm{D}_{14}[\mathrm{x}, \mathrm{y}]\right)\right]
$$

Suppose we could not find $\mathrm{D}_{15}(\mathrm{x})$ or any other s uitable derived predicate. We then decompress $P_{21}$ (A) one layer to obtain

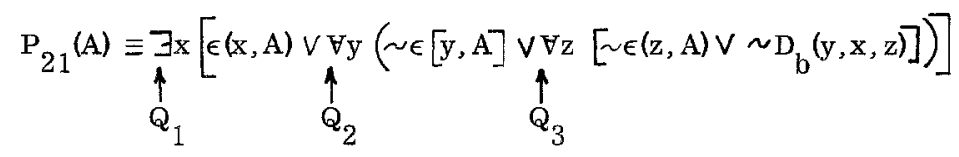

Suppose we now find among our derived predicates

$$
D_{16}(x, y) \equiv \forall z\left[\sim \epsilon(z, A) \vee \sim D_{b}(y, x, z) \vee D_{a}(x, z)\right]
$$

and $\mathrm{E}\left(\mathrm{D}_{16}(\mathrm{x}, \mathrm{y})\right) \approx \frac{\mathrm{K}}{3}_{3}$. The result of our mutation yields:

$$
\breve{P}(\mathrm{~A}) \equiv \exists \mathrm{x}\left[\epsilon(\mathrm{x}, \mathrm{A}) \vee \forall \mathrm{y}\left(\sim \in[\mathrm{y}, \mathrm{A}] \vee \forall \mathrm{z}\left[\sim \epsilon(\mathrm{z}, \mathrm{A}) \vee \sim \mathrm{D}_{\mathrm{b}}(\mathrm{y}, \mathrm{x}, \mathrm{z}) \vee \mathrm{D}_{\mathrm{a}}(\mathrm{x}, \mathrm{z})\right]\right)\right]
$$

(which formula has the same significance as that resulting from our first mutation). If instead of $\mathrm{D}_{16}(\mathrm{x}, \mathrm{y})$, we found among our derived predicates

$$
D_{17}(x) \equiv \forall y\left(\sim \in[y, A] \vee D_{a}[x, y] \vee \forall z\left[\sim \epsilon(z, A) \vee \sim D_{b}(y, x, z) \vee D_{a}(x, z)\right]\right)
$$

and $\mathrm{E}\left(\mathrm{D}_{17}(\mathrm{x})\right) \approx \frac{\mathrm{K}}{2}_{2}$, our mutation of type $5-$ Section 12 yields

\footnotetext{
${ }^{1}$ Use definition of "betweeness" in Example 1, p. 83
} 


$$
\breve{P}(A) \equiv \exists x\left[\epsilon(x, A) \vee \forall y\left(\sim \epsilon[y, A] \vee D_{a}[x, y] \vee \forall z\left[\sim \epsilon(z, A) \vee D_{b}(y, x, z) \vee D_{a}(x, z)\right]\right)\right] .
$$

Suppose no suitable derived predicate is found after examining decompressed forms of the various distributive forms of $P_{21}$, but we discover among our predicate forms

$$
\mathrm{S}_{10}\left(A, K_{1}[\mathrm{x}, \mathrm{y}], \mathrm{K}_{2}[\mathrm{x}, \mathrm{y}]=\exists \mathrm{x}\left[\epsilon(\mathrm{x}, \mathrm{A}) \vee \forall \mathrm{y}\left(\sim \in[\mathrm{y}, \mathrm{A}] \vee \mathrm{K}_{1}[\mathrm{x}, \mathrm{y}] \vee \mathrm{K}_{2}[\mathrm{x}, \mathrm{y}]\right)\right] .\right.
$$

We first note that $D_{14}(x, y)$ has the same variables as $K_{2}(x, y)$ and hence $D_{14}(x, y)$ replaces $\mathrm{K}_{2}(\mathrm{x}, \mathrm{y})$. Then, if $\mathrm{D}_{\mathrm{a}}(\mathrm{x}, \mathrm{y})$ is the most reinforced derived predicate with two free variables, $D_{a}(x, y)$ replaces $K_{1}(x, y)$ and our mutations will yield the same result as our first mutation in this example. (The reader may find it amusing to find models of the formulae resulting from the above mutations,) Possible mutation operations are too numerous for extensive illustration here - see Rothenberg (1973b).

We now consider case (c) of the previous section where $R_{t} \in \bar{C}$ strongly satisfies $P_{i}$. We wish this not to be the case and hence we consider a relaxing mutation if $R_{q} \in \bar{K}$ fails to satisfy $P_{i}$ and a restricting mutation if $R_{q}$ satisfies $P_{i}$. If $R_{q}$ strongly satisfies $P_{i}$ both strategies are unlikely to be successful. In all these cases a neutral mutation should be considered. This also applies when both $R_{q}$ and $R_{t}$ fail to satisfy $P_{i}$. When modified quantifiers are evaluated, in all cases both universal and existential quantifiers are modified and values of $\overline{\mathrm{K}}_{\mathrm{p}}$ and $\hat{\mathrm{K}}_{\mathrm{p}}^{(\mathrm{k})}$ are computed for all such quantifiers. Suppose a neutral mutation of $P_{i}$ is appropriate, and within the scope of $Q_{p}^{(k)}$ (with subformula $F_{p}^{(k)}$ ) there is another quantifier, $Q_{q}^{(k)}$ (with subformula $F_{q}^{(k)}$ ) such that $Q_{p}$ is a universal quantifier and $Q_{q}$ is existential (or vice vers a). Then, if $\bar{K}_{p} \approx \bar{K}_{q}$, a neutral mutation of $F_{p}^{(k)}$ which does not alter the ease of satisfaction of $F_{p}^{(k)}$ is appropriate. In general, a comparison of $\bar{K}_{p}$ and $\overline{\mathrm{K}}_{\mathrm{q}}$ will indicate the degree to which (if any) and in which way a neutral mutation should alter the ease of satisfaction of $\mathrm{F}_{\mathrm{p}}^{(\mathrm{k})}$. The extent to which such a mutation may alter the overlap (see Section 10) of $P_{i}$ and the feature, $\breve{P}_{i}$ (which results from the mutation), without disturbing $E\left(P_{i}\right)$ (i. e. , such that $E\left(P_{i}\right) \approx E\left(\breve{P}_{i}\right)$ is indicated by $\min \left(\bar{K}_{p^{\prime}} \bar{K}_{q}\right)$. In place of a neutral mutation, a "composite mutation" which combines a relaxing and restricting mutation may be used. These will not be discussed here (see Rothenberg, 1973b), but some indications as to the general scheme are shown: The required overlap of $P_{i}$ and $P_{i}$ when a neutral or composite mutation is performed may be determined by examination of the measure of success, $\theta\left(P_{i}, \bar{K}, \bar{C}\right)$ (see Section 10$)$. Neutral mutations are chosen by comparison with predicate forms (somewhat as in step (5) above): If the order of variables in a dummy predicate in a predicate form (which matches $P_{i}$ as in step (5)) differs from the order of variables in a corresponding subformula of $\mathrm{P}_{\mathbf{i}}$ to be mutated, the ordex of variables in that subformula 
is altered to match those of the dummy predicate. If a predicate form differs from $P_{i}$ in the identity of a corresponding subformula (to the subformula to be mutated), the subformula of $\mathrm{P}_{i}$ is replaced by that of the predicate form.

Note that when, for some feature, $P_{i}, F_{p}^{(k)} \equiv F^{(k)} \wedge F_{r}^{(k)}, \hat{K}_{p}$ will estimate $E\left(F_{q}^{(k)} \wedge F_{r}^{(k)}\right)$ while $\hat{K}_{q}^{(k)}$ and $\hat{K}_{r}^{(k)}$ will estimate $E\left(F_{q}^{(k)}\right)$ and $E\left(F_{r}^{(k)}\right)^{p}$ respectively. If $F_{q}^{(k)}$ and $\mathrm{F}_{\mathrm{r}}^{(\mathrm{k})}$ become derived predicates, we will be able to estimate the "orerlap" of $\mathrm{F}_{\mathrm{q}}^{(\mathrm{k})}$ and $\mathrm{F}_{\mathrm{r}}^{(\mathrm{k})}{ }^{\mathrm{r}}$. This is useful in selecting neutral mutations of a feature which replace a subformula which is identical to $F_{q}^{(k)}$ by one which is identical to $F_{r}{ }_{r}^{(k)}$ in such fashion as to control the overlap between the original and mutated feature.

In general, we compute a $\overline{\mathrm{K}}_{\mathrm{p}}$ and $\overline{\overline{\mathrm{K}}}_{\mathrm{p}}$ for each quantifier of a featuxe and for each $R_{\dot{i}} \in \bar{K}$ and $R_{j} \in \bar{C}$. Hence these values of modified quantifiers should properiy be denoted " $\frac{\mathrm{K}}{\mathrm{K}}\left(\mathrm{R}_{\mathrm{q}}\right)^{\prime \prime}$ and " $\overline{\overline{\mathrm{K}}}\left(\mathrm{R}_{\mathrm{q}}\right)^{\prime \prime}$ where $\mathrm{R}_{\mathrm{q}}$ represents the region substituted for the free variable, $A$, in $P_{i}(A)$ when these values are computed. When selecting a mutation, properties of the distributions of $\overline{\mathrm{K}}_{\mathrm{p}}\left(\mathrm{R}_{\mathrm{q}}\right)$ and $\overline{\mathrm{K}}_{\mathrm{p}}\left(\mathrm{R}_{\mathrm{q}}\right)$ over all $\mathrm{R}_{\mathrm{q}} \in \overline{\mathrm{K}}$ and over all $\mathrm{R}_{\mathrm{q}} \in \overline{\mathrm{C}}$ are considered (averages are seldom used-their use was mainly for illustrative purposes). Of particular importance is $\min \left(\bar{K}_{p} \mid R_{q} \in \bar{K}\right)$ and $\max \left(\bar{K}{ }_{p} \mid R_{q} \in \bar{C}\right)$ when $Q_{p}$ is universal and $\max (\bar{K} \mid R \in \bar{K})$ and $\min \left(\bar{K}_{p} \mid R_{q} \in \bar{C}\right)$ when $Q_{p}$ is existential. We are attempting to make the satisfaction gap, $\mathrm{G}\left(\mathrm{P}_{\mathrm{i}}\right)$, as large as possible. Particular properties of the distributions of $\overline{\mathrm{K}}_{\mathrm{p}}(\mathrm{R}$ ) and $\overline{\bar{K}}_{p}\left(R_{q}\right)$ corollate with our measures of success and with $G\left(P_{i}\right)$. Although the detailed strategy for mutation choice is too detailed for description here (see Rothenberg, 1973b), many of its properties can be deduced by the reader from the preceding discussion. Note also that $\overline{\bar{K}}_{p}\left(R_{q}\right)$ differs from $\bar{K}_{p}\left(R_{q}\right)$ in that it is based upon strong satisfaction. The condition for success that $R_{q} \in \bar{C}$ may satisfy $P_{i}$, but not strongly, elucidates the use of $\bar{K}_{p}\left(R_{q}\right.$ ) in the mutation choice procedure. Also of use in selecting neutral mutations is that it has been shown (Rothenberg, 1973b) that:

(15.1) A neutral mutation on a compressed formula (for a feature) is more likely to reduce the overlap betw een the feature and its mutant then a similar mutation on a subformula which is exposed by decompressing the formula one (or more) layers.

\section{Initial Features}

The system retains three lists (of fixed maximum length) of formulae: (1) features, (2) atomic predicates and derived predicates and (3) predicate forms. Those with lowest reinforcement (except atomic predicates) are eliminated when the computer memory allocation for these lists fill (except when complete abstraction is performed and the predicate hierarchy is rebuilt). 
In most applications the trainer will insert any prior knowledge he has of the problem by the direct insertion (at any time in the learning procedure) of derived predicates and/or features.

When no initial features or derived predicates are provided by the trainer, the initial features are generated as follows: an atomic predicate is selected (at random) and is combined with quantifiers and set membership primitives to form formulae which both satisfy the syntactic requirements for features and survive the elimination procedure. If a single primitive does not suffice, a conjunction or disjunction of several is used. As few prim itives as possible are used. Using the primitives for Example 1, such initial features might be:

$$
\begin{aligned}
& \mathrm{P}_{19}(\mathrm{~A})=\exists \mathrm{x} \exists(\psi[\mathrm{x}, \mathrm{y}] \wedge \forall \mathrm{z}[\sim \in(\mathrm{z}, \mathrm{A}) \vee<(\mathrm{x}, \mathrm{z}, \mathrm{x}, \mathrm{y})]) \quad \text { (strongly satisfied by circular } \\
& \mathbf{P}_{20}(\mathrm{~A})=\exists \mathrm{x} \exists \mathrm{y}(\psi[\mathrm{x}, \mathrm{y}] \wedge \mathrm{vz}[\sim \in(\mathrm{z}, \mathrm{A}) \vee<(\mathrm{z}, \mathrm{x}, \mathrm{z}, \mathrm{y})]) \quad \text { (strongly satisfied by linear } \\
& \text { partitions of U) }
\end{aligned}
$$

At the start of the generation procedure $\psi(x, y, \ldots)$ is used for all variables (as above) to prevent contradictory or tautologous features or features with excessively high or low eases of satisfaction.

Consider features which are of the following compressed form wherein $D(x)$ contains no set membership primitives:

$$
\begin{array}{ll}
\mathbf{P}_{1}(A) \equiv \nabla x[\epsilon(x, A) \vee D(x)] & (\equiv \forall x[\sim \epsilon(x, A) \rightarrow D(x)]) \\
P_{2}(A) \equiv \forall x[\sim \epsilon(x, A) \vee D(x)] & (\equiv \forall x[\epsilon(x, A) \rightarrow D(x)]) \\
P_{3}(A) \equiv \exists x[\epsilon(x, A) \wedge D(x)] & \\
P_{4}(A) \equiv \exists x[\sim \epsilon(x, A) \wedge D(x)] &
\end{array}
$$

Notice that all realizations of $P_{2}(A)$ also satisfy $P_{1}(A)$ because of the strong satisfaction requirement. ${ }^{1} \mathrm{P}_{1}(\mathrm{~A})$, however, is strongly satisfied by all data spaces. (Note that it may not be satisfied by subsets of a data space and is hence not a tautology.) $P_{3}$ (A) is strongly satisfied by the null set or an entire data space. $P_{4}(A)$ is strongly satisfied either by the null set or by an entire data space with one point removed.

Clearly, at the beginning of the feature generation procedure, we wish to avoid features with very high or low ease of satisfaction (so that some measure of success is probable). Hence we choose features with compressed form similar to $P_{2}(A)$ when generating initial features. More refined restrictions can be deduced (see Rothen berg, 1973b)

\footnotetext{
$1^{1}$ The above statements may be intuitively illustrated by interpreting "D(x)" as being satisfied only by points on the boundary of the data space.
} 
In general, if a mutation results in a tautology, the tautology is restricted, and if the mutation results in a contradiction, it is relaxed. ${ }^{1}$ In initial feature generation, the following order of mutations is used, which approximates an ordering by the probability of decreasing the overlap of a feature and its mutant:

\section{$\underline{\text { Restricting Mutations }}$}

first: add $+\psi\left(\mathrm{x}_{1} \ldots \mathrm{x}_{\mathrm{n}}\right)^{\prime \prime}$ to the formula by a conjunction (to the innermost phrase) where $\left\{x_{1} \ldots x_{n}\right\}$ includes all bound point variables.

second: add" $\epsilon\left(x_{i}, A\right)$ " or " $\sim \epsilon\left(x_{i}, A\right) "$ to a phrase by a conjunction. This is one phrase at a time from the innermost phrase outward (i takes on appropriate values) - see example in Section 19.

\section{Relaxing Mutations}

first: eliminate " $\psi\left(\mathrm{x}_{1} \ldots \mathrm{x}_{\mathrm{n}}\right)$ from the formula. second: add " $\sim \psi\left(\mathrm{x}_{1} \ldots \mathrm{x}_{\mathrm{n}}\right)$ " to the innermost phrase of the formula by a disjunction (where $\left\{x_{1} \ldots x_{n}\right\}$ includes all bound variables).

third: add " $\in\left(x_{1} A\right)$ " or " $\sim \epsilon\left(x_{i}, A\right)$ to a phrase by a disjunction. This is done one phrase at a time, from the innermost phrase outward.

\section{Neutral Mutations}

first: negate a subformula (from the innermost phrase outward). second: permute the variables in a subformula.

Also, in the initial generation of features, a selection weighting function assigns somewhat higher probabilities of selection to mutations which increase the length of formulae. (Such selection weighting function later prevents the genexation of formulae with an excessive number of variables.) As soon as more than one successful feature is obtained, simple and relation abstractions are performed.

Note also that initial derived predicates of any given number of free variables (when such are required by the mutation procedure and are scarce) may be generated by combining atomic or derived predicates; e.g., $D_{1}(x, y, z) \equiv D_{a}(x, y) \wedge D_{a}(y, z) ; D_{2}(x, y) \equiv \exists z\left(D_{b}[x, y, z]\right)$. 17. Topology ${ }^{2}$

Because of the use of quantified variables in features (rather than constants-i.e., the propositional calculus), each feature has many models (i.e., "generalization" - see Section 1 This applies to tautologies and contradictions which survive the elimination procedure.

2 The author wishes to express his gratitude to Prof. John Myhill of Leeds University and to Prof. Bernard Jaulin of the University of Paris for their suggestions that a topological approach would prove fruitful. 
1, occurs). Functions may exist which map models of the same feature into each other - i.e., the data space must contain certain symmetries. In general, it is desirable that the satisfaction of formulae by regions of the data space be invariant with respect to the transformations of such regions by "translation", "rotation", "reflection", "dilation" (i e., "magnification"), and "contraction" (i.e, "reduction"). It is convenient to impose a topology on the data space so that this will hold and so that the above transformations may be defined. Also, contraction (the inverse of dilation) can vastly reduce the number of points in the data space (and each of its regions). The application of our techniques to such a space with a reduced number of points can result in enormous computation reduction and, in fact, the practicality of the procedures developed rest upon the utilization of such "reduction mappings" (to be defined). The topology described below also relates the finite spaces here employed to the infinite spaces which they approximate and with which model theory customarily deals: (See appendix (p. 124) for the motivation for the definitions below.)

$$
\left(S, n_{s}\right) \in \Gamma \quad \text { (i.e., is a graphic space iff } \quad\left(S=\text { space, } n_{s}=\begin{array}{c}
\text { neighborhood } \\
\text { system })
\end{array}\right.
$$

(a) $\mathrm{S}$ is a V-space (A Frechet space - see Sierpinsky, 1952) with > 1

(b) Each point has a minimum neighborhood element

(c) Each minimum neighborhood has $\geq 2$ points (a consequence of (d) )

(d) $\phi$ and $S$ are the only closed (or open) sets (" $\phi$ " here denotes the null set)

Let each point be defined as "adjacent" to all points in its minimum neighborhood. This induces a metric; i.e., the graph metric derived from the number of edges in the shortest path (along adjacent points in the graph) from one point to another. Write "xy< $\mathrm{zW}^{\text {", eto. }}$ for that metric. (Note that, because of (d) above, $S$ is connected, but not necessarily finite.)

$$
(S, n) \in \hat{\Gamma} \quad \text { (is a graphable space) iff }
$$

$(17.1)(\exists P)\left(\exists n_{p}\right)\left(\exists \phi: n_{p} \rightarrow n_{s}\right)\left[\left(P, n_{p}\right) \in \Gamma\right) \wedge\left(s=U\left\{\phi N \mid N \in n_{p} \wedge N\right.\right.$ is minimal $\left.\}\right) \wedge$

$$
\left.\left(\forall N_{i}, N_{j} \in n_{p}\right)\left(N_{i} \varsubsetneqq N_{j} \rightarrow \phi N_{i} \varsubsetneqq \phi N_{j}\right)\right]^{1}
$$

Given an ordering, $\leq_{0}$, of all elements of $\mathrm{s}^{2}$ and of $\mathrm{P}^{2}$, we define (where $\epsilon$ is an element of $\mathrm{S} \times \mathrm{S})$

\footnotetext{
1 The latter condition is added in order to preserve the intuitive notion. Also, possibly converge by neighborhoods rather than minimum neighborhoods is sufficient. Note that " $P$ " here denotes a space, not a feature (features are always subscripted, e.g. , "P ${ }_{i}$ ").
} 


$$
\mathrm{xy}-\mathrm{zw} \leq_{0} \epsilon \equiv \mathrm{xy} \leq_{0} \mathrm{zw} \vee \exists \vee\left[\left(\mathrm{xv} \leq_{0} \mathrm{zw} \wedge \mathrm{vy} \leq_{0} \epsilon\right) \wedge\left(\mathrm{xy} \leq_{0} \mathrm{zv} \wedge \mathrm{wv} \leq_{0} \epsilon\right)\right]
$$

and so 1

$$
\mathrm{xy}-\mathrm{zw}>_{0} \epsilon \equiv \mathrm{xy}>_{0} \mathrm{zw} \wedge \nabla v\left[\left(\mathrm{xv} \leq_{0} \mathrm{zw} \rightarrow \mathrm{vy}>_{0} \epsilon\right) \wedge\left(\mathrm{xy} \leq_{0} z v \rightarrow \mathrm{wv}>_{0} \epsilon\right)\right]
$$

$\left(\mathrm{P}, \mathrm{n}_{\mathrm{p}} \leq_{0}\right) \in \Gamma_{\mu}(\epsilon)$ (is a uniform graphic space) iff $\left(\mathrm{P}, \mathrm{n}_{\mathrm{p}}\right) \in \Gamma$ and $(\forall \mathrm{x}, \mathrm{y}, \mathrm{z}, \mathrm{u} \in \mathrm{P})$

$$
\left(x y-z u>_{0} \in \rightarrow x y>_{G} z u\right)
$$

(and so $\left.x y \leq_{0} z u \wedge x y>_{0} z u \rightarrow \exists v\left[\left(x v \leq_{0} z u \wedge v y \leq_{0} \epsilon\right) \vee\left(x y \leq_{0} z v \wedge u v \leq_{0} \epsilon\right)\right]\right)$

$\left(P, n_{p}\right)$ is a basis for $S$ (given $\leq_{0}$ ) iff (where $\delta$ is an element of $\left.S \times S\right)$ : P,S satisfies (17. 1) for some $\phi$ and

$$
(\exists \psi: S \rightarrow P)(\exists \delta)(\forall x, y, z, w \in S)\left[\left(x y-z w>_{0} \delta\right) \rightarrow(\psi x, \psi y)>>_{G}(\psi z, \psi w)\right]
$$

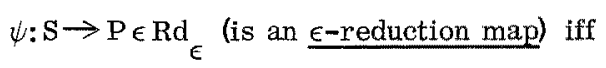

(a) $\psi$ is continuous (i.e., $\psi\left(\mathrm{E}^{\prime}\right) \subset \psi(\mathrm{E}) \cup(\psi(\mathrm{E}))$ ' for all $\mathbf{E} \subset \mathrm{S}$, where $E^{\prime}$ is the derived set of $\left.E\right)^{2}$, and onto

(b) $s \in \Gamma_{u}(\epsilon)$

(c) $\mathrm{P}$ is a basis for $\mathrm{S}$ (i.e., 17.2 holds with the same $\psi$ and with $\delta=\epsilon)$

(d) Card $\mathrm{P}<$ card $\mathrm{S}$.

The following observations are germane in graphable spaces (all references are to

Sierpinsky, 1952):

(a) " $x$ is a limit point of $A$ " means that $x$ is adjacent to an element of $A$ (p. 3). $E^{\prime}$ (the derived set) consists of all non-isolated points of $E$, plus the neighboring points of $E$ (p. 3).

(b) Only the whole space is closed or open but relative closure and openness makes sense (pp. 4,6,15): E C F is closed in $F$ if it is separated from the rest of F by a "white band". Relative open is the same as relative closed.

(c) "Dense in itself" here means no isolated points (p. 13).

\footnotetext{
${ }^{1}$ Note that our definition can be weakened to include the case where $(x y<z w \quad y x<z w)$ (see Rothenberg, 1973b): $\mathrm{xy}-\mathrm{zw} \leq_{0} \epsilon=\mathrm{xy} \leq_{0} \mathrm{zw} \vee \exists \vee\left[\left(\mathrm{xy} \leq_{0} \mathrm{zw} \wedge v \mathrm{y} \leq_{0} \epsilon\right) \vee\right.$ $\left.\left(\mathrm{xy} \leq_{0} \mathrm{z} \vee \wedge \mathrm{wv} \leq_{0} \epsilon\right) \vee\left(\mathrm{vy} \leq_{0} \mathrm{zw} \wedge \mathrm{xv} \leq_{0} \epsilon\right) \vee\left(\mathrm{xy} \leq_{0} \mathrm{vw} \wedge \mathrm{vz} \leq_{0} \epsilon\right)\right]$.

2 Condition (a) may be deducible from the other conditions (see T22, p. 25 of Sierpinsky, 1952, and definition 17. 1 here).
} 
(d) "Scattered" means only isolated points (p. 13).

(e) Two sets are "separated" if a white band separates them (p. 16).

(f) "Connected in the topological sense coincides with the geometrical sense (p. 16).

(g) "Frontier" of $A$ is points of $A$ adjacent to points not in $A$, plus points not in $A$ next to points in A (page 19 - hence corollary on same page).

(b) "Continuous mapping" (from one discrete space to another) means two neighboring points go into neighbors or into the same point.

(i) "Bicontinuous map" (homomorphism) means " $\mathrm{x}$ next to $\mathrm{y}$ iff $\mathrm{f}(\mathrm{x})$ next to $\mathrm{f}(\mathrm{y})$ (p. 28).

Reduction maps are continuous maps and all properties preserved under continuous maps are preserved by reduction maps; i.e., connectedness (but not disconnectedness) boundary connected (i.e. , simply connected), frontiers are preserved (but boundaries are not), etc.

The transformations of "contraction" and "dilation are defined by reduction maps and their inverses. "Rotation", "translation" and "reflection" are defined (together) by a biunique mapping from a space $\mathrm{S}$ to a transformed space, $\mathrm{P}$, such that (a), (b) and (c) (but not (d)) of (17.3) are satisfied. The following have been proved (Rothenberg, 1973b) where $\psi: \mathrm{S} \rightarrow \mathrm{P} \in \mathrm{Rd} \mathrm{E}_{\epsilon}$ and where each feature, $\mathrm{P}_{\mathrm{i}}$, is constructed using the atomic predicate, $"<(\mathrm{x}, \mathrm{y}, \mathrm{z}, \mathrm{w}) ":$

If $\mathrm{S}=\left|\mathrm{M}\left(\mathrm{P}_{\mathbf{i}}\right)\right|$, then $\mathrm{P}=\left|\mathrm{M}\left(\mathrm{P}_{\mathbf{i}}^{*}\right)\right|$ where $\mathrm{P}_{\mathbf{i}}^{*}$ is the same formula as $\mathrm{P}_{\mathbf{i}}$ except that its degree of satisfaction has been altered by a bounded amount which is a function, $f$, of $\epsilon$ and of the syntax of $\mathbf{P}_{\mathbf{i}}-\mathbf{i}$. e., in general (see (14.1):

$$
\left|D\left(P_{i}, S\right)-D\left(P_{i}, P\right)\right| \leq f(\epsilon, n) \quad \begin{gathered}
\text { (note again that } P \text { is a space, } \\
\text { not a feature like } \left.P_{i}\right)
\end{gathered}
$$

where $\mathrm{n}$ depends upon the number of occurrences of the atomic predicate " $<(\mathrm{x}, \mathrm{y}, \mathrm{z}, \mathrm{w})^{\prime \prime}$ in the subformula of $P_{i}$ within the scope of the relevant (to $D\left(P_{i}, P\right)$ ) modified quantifier. Actually $f$ depends upon other factors as well, but minimally so when the preimage of each point $p_{i} \in P$ is chosen so that $\epsilon$ is minimal over all of $s^{4}$. (In this case note that if $s$ is homogeneous, the number of points in the preimage of each $p_{1} \in \mathbf{P}$ is determined by $\epsilon$.) When $\mathrm{S}$ is, for example, a bounded rectangle in Euclidean space, and $P$ is finite, $f=K / \operatorname{card}(P)$ where $K$ is a function of $n$ and specific metric properties of $S$, and $\operatorname{card}(P)$ is a function of $\epsilon . \quad($ Note: as $\epsilon \rightarrow 0, f \rightarrow 0$.

Let $S$ be finite. Suppose $R \subset S$ and $\bar{R}$ is the image of $R$ in $P$ under the above reduction mapping (henceforth called the degraded image of $R$ ). Let each $p_{j} \in \psi(R)$ such 
that the preimage of $p_{j}$ contains elements both of $R$ and $S-R$ be called a dubious point ${ }^{1}$ denoted by " $p_{j} \in d(\bar{R})$ ". Then

$$
\left|\mathrm{D}\left(\mathrm{P}_{\hat{i}}, \mathrm{R}\right)-\mathrm{D}\left(\mathrm{P}_{\mathrm{i}}, \overline{\mathrm{R}}\right)\right| \leq \mathrm{g}(\epsilon, \mathrm{n}, \mathrm{m})
$$

where $m=\operatorname{card}\left(p_{j} \mid p_{j} \in d(\bar{R})\right) / \operatorname{card}\left(p_{j} \mid p_{j} \in \bar{R}\right)$ and the above remarks (in (17.4)) about $f$ also apply to $\mathrm{g}$. Larger values of $\mathrm{m}$ result in larger values of $\mathrm{g}$. (Note: if $\mathrm{R}$ is a randomly chosen connected region in a uniformly sampled place, $m$ is approximately equal to its circumference divided by its area.)

Similar (but tighter) bounds exist when $\psi$ executes a translation, rotation or reflection.

Note the similarity between the left hand portions of the formulae $(17.4)$ and $(17.5)$ and the definition (14.2) of the satisfaction gap of $P_{i}, G\left(P_{i}\right)$. Hence the following application of (17.4) and (17.5) is appropriate:

Normally the data space, $U$, is uniformly sampled - along a square grid. ${ }^{2}$ The image of $U$ under a reduction map is similar, but is a smaller (i.e., fewer points) space. When $G(P)>0$ we may (from $G\left(P_{i}\right)$ ) determine an $\epsilon$ (and select a corresponding reduction mapping) such that the application of $P_{\mathfrak{i}}$ to the degraded regions in $P$ still results in $G\left(P_{i}\right)>0$ (see remarks following $(17.4)$ ). For such $\epsilon$ no information relative to the satisfaction of $P_{i}$ is lost by application of the reduction map. Since $f \approx K / \operatorname{card}(P)$ (see above) and card(P) decreases with $\epsilon$, a reduction map with a small $\epsilon$ (i.e., card(P) is large) will reduce $G\left(P_{i}\right)$ by a small amount. However, the amount of calculation reduction that can result from the application of such a reduction mapping is enormous. In addition to uses when $G\left(P_{i}\right)>0$, the evaluation of $P_{i}$ in a relatively large space may be avoided by confining such evaluation to regions which are preimages of regions in smaller spaces where models of $\mathrm{P}_{i}$ have been found. (That is, the evaluation of a feature in a smaller space precedes that in a larger, the former with a lower required degree of satisfaction than the latter. Evaluation in the larger space is then performed in that restricted subspace which is the preimage of a model found in the smaller.)

Other methods of computation reduction are used. Regions of the sample space which satisfy (or fail to satisfy) certain predicates have their identifications stored together with the predicate abbreviations. Then "do loops" in formulae range only over those regions which are already known to satisfy subpredicates included within these loops. Further

1 Data dependent unary atomic predicates (e.g., $\overline{\mathrm{D}}(\mathrm{x})$ in Example 1) can be included by extending the definition of dubious point to include any $p_{i} \in /(R)$ whose preimage contains elements with differing values of the unary atomic predicate. Then (17.5) still applies. 2 Note: a sampling along the centers of a covering of $\mathrm{U}$ by regular hexagonal tiles would permit a smaller choice of $\epsilon$ in a reduction map. 
computation reduction is accomplished by the use of implications between features (these are known across all but neutral mutations - see Section 12) to avoid unnecessary testing. A similar technique may be employed to minimize search and storage when data independent primitives are related by implication ("tree searches" are used).

Note also that the data independent portions of features, $P_{i}^{I}$, are always evaluated in either some $R_{j}$ or $\left|M_{j}^{(U)}\left(P_{i}^{D}\right)\right|$ (see Section 13), never in the entire data space. In this manner enormous computations are avoided. Furthermore, $P_{i}$ is evaluated in $s$ by utilizing the properties of nested normal form to remove points from the space until a model (i.e., strong satisfaction) is encountered. Roughly (see Rothenberg, 1973b), if $\mathrm{s}_{\neq}\left|\mathrm{M}_{j}^{(U)}\left(\mathrm{P}_{\mathrm{i}}\right)\right|$ then $S=\left|M_{j}^{(U)}\left(\sim F_{j}\right)\right|$. Then points are removed, one at a time so that $\sim P$ is violated as soon as possible.

\section{Hierarchy of Representation}

Here the size of the problem is reduced (and its representation changed) by the use of hierarchical levels wherein objects are expressed as collections of subobjects, etc. , all such objects being defined by features which are similaxly hieraxchically organized. At each such hierarchical level the techniques previously discussed are independently applied.

All "atomic predicates" ("primitives"), "features", "points", "objects" and "object classes" (see Section 2) will now have "first level" prefixing their names. Also the following restrictions are added to our syntax:

(18.1) (a) For each first level feature, $P_{i}(A)$, which begins with a string of existentially quantified set variables (e.g., " $\exists \mathrm{B} \exists \mathrm{C} \exists \mathrm{D}, . . \mathrm{t})$, those set variables $(B, C, D, \ldots)$ are allowed to range only over models of other features already in the descriptive basis (i.e., objects).

(b) Furthermore, the above set variables may not be identical with the region that is substituted for the free variable, $A$, in $P_{i}(A)$ above (i.e., $\sim(\mathrm{A}=\mathrm{B}) \wedge \sim(\mathrm{A}=\mathrm{C}) \wedge \sim(\mathrm{A}=\mathrm{D}) \ldots)$.

Also the following, called the identification measure is added to our measures of success (see Section 10) and is used when generating features:

$$
\bar{\theta}\left(\mathrm{P}_{\mathrm{i}}, \overline{\mathrm{K}}, \overline{\mathrm{C}}\right)=\gamma\left(\mathrm{P}_{\mathrm{i}}, \overline{\mathrm{K}}\right)+\gamma\left(\mathrm{P}_{\mathrm{i}}, \overline{\mathrm{C}}\right)
$$

Whenever we advance a level (from the $\mathrm{n}^{\text {th }}$ to the $\mathrm{n}+1$ st level) the following operations are performed (see Example 7 which follows).

(18.3) (a) All $\mathrm{n}^{\text {th }}$ level objects, $A_{1}, A_{2} \ldots A_{r}$ become $n+1$ lst level points,

$$
\mathrm{p}_{1}, \mathrm{p}_{2}, \ldots, \mathrm{p}_{\mathbf{r}} \text {. }
$$


(b) All $n^{\text {th }}$ level features, $P_{1}(A), P_{2}(A), \ldots P_{m}$ (A) become $\underline{n+1 \text { st level }}$ primitives (atomic predicates), $\alpha_{1}(\mathrm{x}), \alpha_{2}(\mathrm{x}), \ldots \alpha_{\mathrm{m}}(\mathrm{x})$.

(c) If $P_{i}(A)$ is an $n^{\text {th }}$ level feature which begins with a string of existentially quantified set variables, i.e. ,

$$
P_{i}(A) \equiv \exists B_{1}, \ldots, \exists B_{j}\left[F\left(A, B_{1}, \ldots, B_{j}\right)\right]
$$

then $\mathrm{F}\left(\mathrm{A}, \mathrm{B}_{1}, \ldots, \mathrm{B}_{\mathrm{j}}\right)$ becomes an $\mathrm{n}+1$ st level primitive $\beta\left(\mathrm{x}_{1}, \mathrm{x}_{2}, \ldots \mathrm{x}_{\mathrm{j}+1}\right)$. (all sets of $n^{\text {th }}$ level objects which together satisfy $F$ are known (see (18.1). Hence the values ("true" or "false") of $F\left(A, B_{1}, \ldots B_{j}\right)$ are known for all arguments and so are the corresponding values of $\beta\left(x_{1}, x_{2}, \ldots x_{j+1}\right)$. This procedure permits both relations between objects and between other relations to be expressed at successive levels-see illustration below and Rothenberg (1973b).

Whenever the following conditions are met we advance a level:

(18.4) For each $R_{i} \in \overline{\mathrm{C}}$ there exists a set of objects $\left\{A_{1}, A_{2}, \ldots A_{n}\right\}$ such that

$$
\bigcup_{k=1}^{n} A_{k}=R_{i}
$$

and

$$
\mathrm{n}<\operatorname{card}\left(\mathrm{R}_{i}\right)
$$

and

(c) There does not exist an $\mathrm{R}_{\mathrm{j}} \in \overline{\mathrm{C}}$ and a set of objects $\left\{\mathrm{B}_{1}, \mathrm{~B}_{2}, \ldots \mathrm{B}_{\mathrm{m}}\right\}$ such that both $\bigcup_{\ell=1}^{m} B_{\ell}=R_{j}$ and all such $B_{\ell}$ (and $n$-tuples of $B_{\ell}$ ) satisfy the same next level atomic predicates as the $A_{k}$ (and n-tuples of $A_{k}$ ) in an $\left\{A_{1}, A_{2}, \ldots A_{n}\right\}$ satisfying condition (a) of (18.4) above. (Note that these atomic predicates include all features and relations constructed therefrom (as in $(18,3)$ above) at the level being examined. This condition assures that there exist no $R_{\dot{I}} \in \bar{K}$ and $R_{j} \in \overline{\mathrm{C}}$ which consist of unions of indistinguishable (by features satisfied) objects.)

The above results in a change of hierarchical level whenever a reduction in the data to be represented results thereby. (Provis ion for trainer intervention when desired exists (see Rothenberg, 1973b).)

The definition of descriptive basis remains the same as in Section 2 except that all features at all levels are included. $\underline{n}^{\text {th }}$ level objects are defined in terms of $n^{\text {th }}$ level points (i.e., n-1st level objects) exactly as first level objects are defined in terms of first level points. Similarly, the definitions of $\mathbf{n}^{\text {th }}$ level features, object classes, etc., are defined as before in terms of the other entities at the same level. However, the definition of $\underline{n}^{\text {th }}$ level primitive is extended to include the atomic predicate, $\epsilon^{(i, k)}\left(x^{(k-i)}, x^{(k)}\right)$ 
wherein $k$ and $i$ are fixed (of course, $k-i<k \leq n+1$ where $n$ is the hierarchical level). This atomic predicate is called recurs ive containment and asserts that there exists a sequence of points at the indicated (by the superscript) levels such that, if we put $x^{(k-i)}$ in front of the sequence and $x^{(k)}$ at the end, each term in the sequence is an element of the next (there are $i+1$ terms in such a sequence). (This allows statements about first level points in second level (and higher level features - see Rothenberg, 1973b).)

\section{Example 7}

Suppose we wish to distinguish photographs (from above) of any number of stacked discs from pictures of any other objects. Then the $R_{i} \in \bar{K}$ will consist of diagrams of the following form:

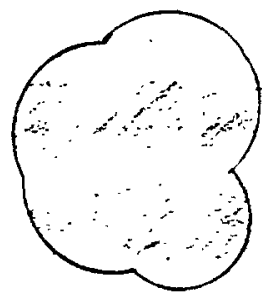

(1)

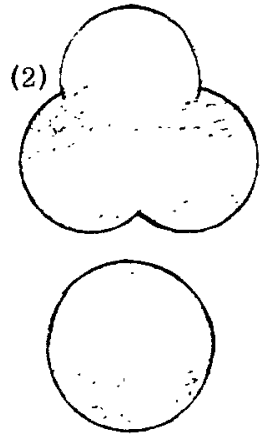

(3)

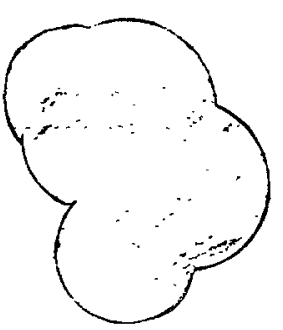

(4)

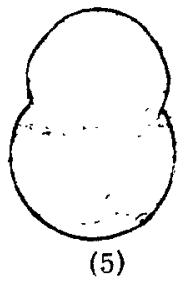

etc.

$R_{j} \in \bar{C}$ will consist of diagrams which cannot be formed by a finite number of overlapping circles. Suppose we have thus far generated feature $P_{i}(A) \equiv D_{d}(A) \wedge$ $\exists \mathrm{x} \exists \mathrm{yVz}(\epsilon(\mathrm{x}, \mathrm{A}) \rightarrow \sim<[\mathrm{x}, \mathrm{y}, \mathrm{x}, \mathrm{z}])$ (see Example 1, Section 1) which is strongly satisfied by dark circular regions and $P_{2}(A)=\exists B \exists x\left(\in[X, A] \wedge \in[x, B]\right.$ ). (Note that $\bar{\theta}\left(P_{2}, \bar{K}, \bar{C}\right)$, see (18.2), is very high in this example.) Note that conditions (18.4) are now met. Hence we advance a level as follows (see (18.3)): (1) All first level objects become second level points, $\mathrm{p}_{1}, \mathrm{p}_{2}, \ldots, \mathrm{p}_{\mathrm{r}}$. (2) $\mathrm{P}_{1}(\mathrm{~A})$ becomes a second level atomic predicate, $\alpha_{1}(\mathrm{x})$ (satisfied by second level points). (3) $\mathrm{P}_{2}$ (A) is transformed by eliminating the existential quantifier at the left of its formula to form $F(A, B)=\exists x(\epsilon[x, A] \wedge \epsilon[x, B])$ which is satisfied by pairs of first level objects (which are now second level points). F(A, B) now becomes a second level atomic predicate, $\beta(x, y)$. We may now, at the second level, construct the second level feature, $\mathrm{P}_{1}^{(2)}(\mathrm{A}) \equiv \forall \mathrm{x}\left(\epsilon[\mathrm{x}, \mathrm{A}] \longrightarrow \alpha_{1}(\mathrm{x}) \wedge \forall \mathrm{y}[\epsilon(\mathrm{y}, \mathrm{A}) \rightarrow \beta(\mathrm{x}, \mathrm{y})]\right)$ which is satisfied only by $R_{i} \in \bar{K}$.

The following definitions should also be added to those of Section 2: 
Description: This is a list of all features at all levels used in the descriptive basis and lists all objects strongly satisfying and satisfying each of these features and the elements of each such object. 1

Filter predicates: (optional) These are formulas of the same form as first level features which must be satisfied by regions of the sample space called infra-objects in order for $s$ ub-regions of these infra-objects to be candidates for becoming first level objects. These may be used to substantially reduce the number of regions which must be considered for satisfaction of a first level feature. Typically, filter predicates might include formulae whose satisfaction guarantees that all points in a region have data values in a specified range and/or that such regions form connected sets. Filter predicates might also be used to eliminate the need for data dependent features.

\section{Flow of the System}

Features are evaluated by whether they are strongly satisfied by the required regions and perform required partitions (as initially specified). Other criteria (in the absence of initially specified objects and partitions) include feedback to a human trainer wherein objects in the description of a picture are projected on a cathode ray tube for comparison with the input picture. Also, at the request of the trainer, such objects may be successively replaced by other regions which satisfy (and strongly satisfy) the same features as these objects, so that the trainer may "see" what the system does not "see" as well as what it does "see" (see Rothenberg, 1973b)). The trainer thereby evaluates the performance of the system, which he indicates by means of positive or negative "feedback".

Note again that the trainer may include any prior knowledge he has of the problem by direct insertion at any time of derived predicates and/or features. The system, in a practical application, thereby begins "learning" as if it had already "learned" all such knowledge provided by the trainer.

When feedback is absent and success has been obtained, the system nonetheless continues to compute, attempting to increase the satisfaction gap, to lessen the number of quantifiers in features and to eliminate unnecessary objects; i.e., those which are in the description, but which are not relevant to the problem as defined initially (i.e., do not correspond to any element of $\overline{\mathrm{K}}$ or $\overrightarrow{\mathrm{C}}$ ) or by the trainer.

Very briefly, the overall flow of the system is as follows (trainer interaction is not included):

\footnotetext{
1 In some applications, if two features at the same level have identical data dependent expressions in their formulae and if an object which strongly satisfies one feature is properly contained in an object which strongly satisfied the other feature, the former object is omitted from the description.
} 
(1) Initial features are either provided by the trainer or constructed as in Sec. 16.

(2) Simple and relation abstraction are performed. (When the lists are full reconstruct the predicate hierarchy and if there are still too many formulae, those with lowest reinforcement are eliminated.)

(3) That feature with the highest reinforcement is selected for mutation.

(4) The mutation is chosen as in previous sections. If the new feature meets syntactic requirements and has a higher measure of success than some other feature (and if the list of features is full), the feature with the lowest measure of success is eliminated and the new feature is added to the list of features.

(5) Is the satisfaction gap $>0$ ? If so, perform reduction map.

(6) Is the satisfaction gap large enough to stop computing? (Predetermined by trainer.)

(7) Are the criteria for advancing a hierarchical level met? If so, advance the level, and return to step (1) at the next level. If not,

(8) Return to step (2).

The procedure is, of course, extremely complex in detail ${ }^{1}$ - see Rothenberg (1973b).

\section{Open Questions}

A characterization of the semantics of predicates which can be constructed from a given set of primitives is required. A partial decision procedure which, as far as possible, avoids the generation of features which are conjunctions of contradictory predicates is needed. The generation of predicates with no finite model should also be avoided where possible (e.g., $P_{i}(A) \equiv \forall x \forall y \exists z\left(\epsilon[x, A] \wedge \epsilon[x, A] \rightarrow\left[\epsilon(z, A) \wedge D_{b}(x, z, y)\right]\right)$ is satisfied by convex sets only when the data space is infinite-otherwise it is vacant). It would be useful to know in advance the minimum depth (of nested phrases) of the most deeply nested predicate needed to separate all inequivalent regions in a data space. ${ }^{2}$ The replacement of "ease of satisfaction" by an easily computed measure which retains the ordering of formulae, $F_{i}\left(b y=\left(F_{i}\right)\right)$, when the data space is infinite is desirable.

Also significant is the question of whether the fraction consisting of the number of logically inequivalent formulae with $\mathrm{n}$ quantifiers divided by the total number of formulae with $n$ quantifiers increases or decreases as $n$ increases. This indicates the chances of skipping and not recapturing a needed feature in a "depth first" search on the tree of fea-

\footnotetext{
1 Actually, a state-space search procedure is used-see Nilsson (1971).

2 Note that a maximum must be set on the number of quantifiers in a feature, both for practical reas ons and to avoid trivial solutions (i.e., no generalization).
} 
tures and is relevant to search strategy (i.e., to proceed by large mutations which change the number of quantifiers or by small mutations??.

The extension of the system to include restricted third-order quantification (over features in lower levels of the descriptive basis) is being considered. This would enable the system to describe objects of similar kind (with respect to the descriptive basis) and would impart to it some self-descriptive power of a limited sort. However, problems arise due to the potential incompleteness of the descriptive basis at any time during the learning procedure.

Another important practical problem is the elimination of features which are trivial (as well as vacant or tautologous) in a particular application (e.g., $P_{i}(A) \equiv$ $\exists x \exists y(\epsilon[x, A] \wedge \in[y, A] \wedge \psi[x, y])$ ). Such methods have already been derived, but the need for additional techniques will doubtless become evident during early experiments using the program.

It appears that for certain choices of primitives (such as those in the examples here) the use of Skolem functions (Schoenfeld, 1967) may result in eliminating existential quantifiers and hence in substantial simplification of the mutation procedure.

Of greatest significance, however, is that it appears that the entire system can be reduced to a first order system by (a) an appropriate choice of primitives (see Beth defineability theorem (Schoenfeld, 1967; Reyes, 1969)) or (b) the development of a procedure for replacing some second order formulae by first order predicates recursively defined. For example, a second order formula asserting that "point $\mathrm{x}$ is connected to point $\mathrm{y}$ " may be replaced by

$$
F(x, y)=\exists z\left(\left[D_{a}(x, z) \wedge D_{a}(z, y)\right] \vee\left[D_{a}(x, z) \wedge F(z, y)\right]\right)
$$

The computation expended in the procedures described increases rapidly as the number of primitives used increases. Hence, the choice of the smalles $t$ number of primitives which are appropriate to a problem and which result in a sufficiently expressive language is of central importance. (Note that the primitive used in our illustrations, $<(x, y, z, w)$, is extremely powerful-e.g. . "xy $+y z=x z$ " can be defined by using $D_{b}(x, y, z)$ and then we may define " $x y+v w=p q$ " by using $x y+y z=x z,=(y, z, v, w)$ and $=(x, z, p, q)$, etc.) When many primitives are required, it is advisable to distribute them among the hierarchical levels used-i.e., so that primitives not at the first level are introduced at the second (e.g. , "card (A)"), etc. Further work on this question could prove to be of considerable practical value.

It should also be noted that although this has not been done in the examples here, it appears profitable to experiment by using the system with primitives specifying (optical) spectral or holographic information. 


\section{Appendix to Section 17 (Topology)}

All references are to Sierpinski's (1952) General Topology, first chapter (which deals with Frechet spaces). First we draw attention to several rather peculiar properties of Frechet spaces which are not topological spaces:

(1) In a Frecet space the sum of two closed sets need not be closed (p. 11, bottom). Hence the intersection of open sets need not be open. [Note that closed sets are sets which contain all their limit elements, p. 6 (4.), and open set is defined as the complement of a closed set, p. 11 (6.), and that the condition "every neighbourhood of an element of $\mathrm{K}$ is an open set" is necessarily true only when $\mathrm{K}$ satisfies the definition of a topological space (p. 38 , middle). See also p. 12, Theorem 5.]

(2) In a Frechet space the condition that "a neighbourhood contains an element" does not imply that "the neighbourhood is a neighbourhood of that element". That is, a neighborhood may contain an element of which it is not a neighborhood (i. e., $x \in N(y)$ and $N(y) \neq N(x)$ ). Note also that conditions $\gamma$ and $\delta$ on p. 38 may not be satisfied-i.e., distinct elements may not be contained in distinct neighborhoods.

Before attempting to explain the motivation for the axiom "The only closed sets are the null set and the universal set", we present an illustration of such a Frechet space:

Let our space, $S$, consist of nine points: $S=\{a, b, c, d, e, f, g, h, i\}$. For convenienc convenience, picture them in a rectangular array:

$$
\begin{aligned}
& \text { a.b.c. } \\
& \text { d.e.f. } \\
& \text { g.h.i. }
\end{aligned}
$$

Let the minimum neighborhood of a point consist of all points which are nearest to it in the above rectangular array (i.e., those points directly above, below, to the right of and to the left of it (if there are such points)). Thus our minimal neighborhoods are:

$$
\begin{aligned}
& N(a)=\{a, b, d\} \\
& N(b)=\{a, b, c, e\} \\
& N(c)=\{b, c, f\} \\
& N(d)=\{a, d, e, g\} \\
& N(e)=\{b, d, e, f, h\} \\
& N(f)=\{c, e, f, i\} \\
& N(g)=\{d, g, h\} \\
& N(h)=\{e, g, h, i\} \\
& N(i)=\{f, h, i\}
\end{aligned}
$$

For all $x$, the neighborhoods of $x$ will be all sets which include $N(x)$. 
Note that all sets except the null and the entire set are neither open nor closed. For example, let $E=\{c, e, f, g, h, i\}$. Let $E^{\prime}$ denote its derived set of $E$ (i.e., the set of all its limit elements). Let $F$ denote the complement of $E(i . e ., S-E)$ and $F^{\prime}$ denote the derived set of $F$. Then $F=\{a, b, d\}$. Note that $b \in E^{\prime}$. Hence $\sim\left(E^{\prime} \subset E\right)$ and $E$ is not closed. Note also that $e \in F^{\prime}$. Hence $\sim\left(F^{\prime} \subset F\right)$ and $F$ is not closed.

The same argument can be repeated for all other non-empty proper subsets of $\mathbf{S}$. Also, $\mathrm{S}$ can be made as large as we wish by adding elements to the rows and columns of the rectangular array. If the minimal neighborhoods are similarly defined all non-empty proper subsets of $\mathrm{S}$ will be neither open nor closed.

Observe that the imposition of the conditions on a Frechet space that "each point have a minimal neighbourhood and that $\phi$ and $S$ be the only closed sets" is consistent with the definition of a minimal neighborhood of a point in a finite space as containing those points "adjacent" to that point. The conditions also prevent the shrinkage of neighborhoods to the trivial case where they contain only one point. This is as might intuitively be expected in a finite space, and the preservation of conditions (a) - (i) of Section 17 is also in accordance with intuition. Also, we are assumed that our entire space is connected so that a graph metric may be imposed (Section 17).

Note that condition $\delta$ on page 38 of Sierpinski does not hold, and hence we do not have a topological space.

Another alternative was available: The condition that " $\phi$ and $S$ are the only closed sets" might have been replaced by "all sets are both open and closed". Then we could have defined the minimal neighborhoods in our above illustration non-uniquely:

$$
\begin{aligned}
& N_{1}(a)=\{a, b\} \quad N_{2}(a)=\{a, d\} \\
& N_{1}(b)=\{b, a\} \quad N_{2}(b)=\{b, c\} \quad N_{3}(b)=\{b, e\} \\
& N_{1}(c)=\{c, b\} \quad N_{2}(c)=\{c, f\} \\
& \mathrm{N}_{1}(\mathrm{~d})=\{\mathrm{d}, \mathrm{a}\} \quad \mathrm{N}_{2}(\mathrm{~d})=\{\mathrm{d}, \mathrm{e}\} \quad \mathrm{N}_{3}(\mathrm{~d})=\{\mathrm{d}, \mathrm{g}\} \\
& N_{1}(e)=\{e, b\} \quad N_{3}(e)=\{e, d\} \quad N_{3}(e)=\{e, f\} \quad N_{4}(e)=\{e, h\}
\end{aligned}
$$

etc.

Now each element would be the intersection of all its neighborhoods. But now condition $\beta$ on page 38 of Sierpinski would not be satisfied and we still would not have a topological space. Of greater importance, how would we now obtain the necessary graph metric.

Also, the imposition of this type of neighborhood system on a finite space seems counterintuitive. 
In summary, the purpose of the topological section of this paper is to impose conditions on the given structure $<\mathrm{S}, \leqslant_{0}>$ so that it could be mapped into a structure with fewer elements, $\langle P, \leqslant\rangle_{\epsilon},{ }^{1}$ such that if a subset, $A$, of $S$ is a model of a formula, $F$, the image, $f(A)$, of $A$ (in $P$ ) is a model of a formula, $\bar{F}$, which is obtained from $F$ by the substitution of a modified quantifier for a universal or existential quantifier in $F$.

$\overline{1} \mathrm{xy} \leq_{\epsilon} \mathrm{zw} \equiv \mathrm{xy}-\mathrm{zw} \leq \leq_{0} \epsilon$ 


\section{BIBLIOGRAPHY}

Addison, J., L. Henkin and A. Tarski (1965), "Theory of Models", Proc. 1963 International Symposium at Berkeley, North Holland Co., Amsterdam.

Amarel, S. (1962), "An Approach to Automatic Theory Formation", in Principles of Self Organization, Trans. Illinois Symposium on Self Organization (Von Foerster and Zopf, Eds.), Pergamon Press, New York.

Banerji, R. (1969), Theory of Problem Solving, An Approach to Artificial Intelligence, American Elsevier Publishing Co., Inc., New York.

Bell, J. and A. Slomson (1969), Models and Ultraproducts, North Holland Co., Amsterdam.

Block, H.D., N.J. Nilsson and R.O. Duda (1964), "Determination and Detection of Features in Patterns", Computer and Information Sciences (J.T. Tou and R. Wilcox, Eds), Spartan Books, $75-110$.

Bongard, M. (1970), Pattern Recognition (J.H. Hawkins, Ed.), Spartan Books

Bremermann, H.J. (unpublished manuscript), "Artificial Intelligence and Neurobiology", Department of Mathematics, University of California, Berkeley.

Ellentuck, E. (1971), "Direct Products of Relational Systems", U.S. Air Systems Command Final Report, Jan. 1, 1971 (see Rothenberg, 1971).

Fogel, L.J., A.J. Owens, and M.J.Walsh (1966), Artificial Intelligence through Simulated Evolution, John Wiley and Sons, New York.

Friedberg, R.M. (1958), "A Learning Machine, Part I", IBM J. Research and Development, $2,2-13$.

Friedberg, R.M., B. Dunham and J.H. North (1959), "A Learning Machine, Part II", IBM J. Research and Development, 3, 282-287.

Hawkins, J. (1970), "Textual Properties of Pattern Recognition", Picture Processes and and Psychopictories (B.S. Lipkin and A. Rosenfeld, Eds), Academic Press, New York, $347-370$.

Hintikka, J. (1953), "Distributive Normal Forms in the Calculus of Predicates" Acta Philos. Fennica, $6,1$.

Julesz, B. (1969), "Cluster Formation at Various Perceptual Levels", in Methodology of Pattern Recognition (S. Watanabe, Ed.), Academic Press, New York, 297-315.

Kolers, P.A. (1970), "The Role of Shape and Geometry in Picture Recognition", Picture Processing and Psychopictorics (B.S. Lipkin and A. Rosenfeld, Eds.), Academic Press, New York, 181-202.

Lee, R.C.R. (1967), "A Completeness Theorem and a Computer Program for Finding Theorems Derivable from Given Axioms", Ph. D. Dissertation, University of California at Berkeley.

Lofgren, L. (1967), "Recognition of Order and Evolutionary Systems", in Computer and Information Science - II (J. Tou, Ed.), Academic Press, New York, 165-175.

Minsky, M. (1963), "Steps Toward Artificial Intelligence", in Computers and Thought (E.A. Feigenbaum and J. Feldman, Eds.), McGraw-Hill Book Co., Inc., New York, $406-450$.

Muerle, J.L. (1970), "Some Thoughts on Texture Discrimination by Computer", Picture Processing and Psychopictorics (B.S. Lipkin and A.Rosenfeld, Eds.), Academic Press, New York, 371-380. 
Nilsson, N. J. (1971), Problem-solving Methods in Artificial Intelligence, McGraw-Hill Book Co., Inc., New York.

Pessin, A. and D. Rothenberg (in preparation), The Weak Second Order Predicate Calculus as a Programming Language.

Reyes, G.E. (1969), "Local Defineability Theory", Annals of Math. Logic, 1, 101.

Rosenfeld, A. and B. Lipkin (1970), "Texture Analysis", in Picture Processing and Psychopictorics (B. Lipkin and A. Rosenfeld, Eds.), Academic Press, New York, 309-347.

Rothenberg, D. (1971), "An Adaptive Linguistic Model for Pattern Representation and Recognition", Final Report, Air Force Systems Command, USAF, Directorate of Mathematical and Informational Sciences, January 1, 1971.

Rothenberg, D. (1973a), "A Pattern Recognition Model Applied to the Perception of Pitch", AFOSR Technical Report, January 1969, revised version to appear as series of articles in Mathematical Systems Theory. See also this volume.

Rothenberg, D. (1973b), "A Theory of Feature Generation", a series of four articles to be submitted to Mathematical Systems Theory (Copies available from Mathematics Department, Rutgers University, University College, New Brunswick, New Jersey).

Schoenfeld, J. (1967), Mathematical Logic, Addison Wesley, Reading, Massachusetts.

Selfridge, O.G. and U. Neisser (1963), "Pattern Recognition by Machine", in Computers and Thought (E.A. Feigenbaum and J. Feldman, Eds.), MeGraw-Hill Book Co., Ine., New York, 237-250.

Sherman, R. and G.W. Ernst (1969), "Learning Patterns in Terms of Other Patterns", in Pattern Recognition, Pergamon Press, New York, (Vol. 1), 301-313.

Sierpinsky, (1952), General Topology, University of Toronto Press, Toronto.

Slagle, J., C. Chang and R. Lee (1969), "Completeness Theorems for Semantic Resolution in Consequence Finding", Proc. International Joint Conference on Artificial Intelligence, May 7-9, 1969, Washington, D.C. (D.E. Walker and J.M. Norton, Eds.), $281-285$.

Solomonoff, R.J. (1964), "A Formal Theory of Inductive Inference - Part I", Information and Control, $7,1-22$.

Solomonoff, R.J. (1964), "A Formal Theory of Inductive Inference - Part $\Pi "$ " Information and Control, $7,224-254$.

Uhr, L. and C. Vossler (1963), "A Pattern Recognition Program that Generates, Evaluates, and Adjusts its Own Operators", in Computers and Thought, (E.A. Feigenbaum and J. Feldman, Eds.), MeGraw-Hill Book Co., Inc., New York, 251-268.

Wang, H. (1960), "Toward Mechanical Mathematics", IBM J. Research and Development, $\underline{4}$, 2-22. 